\title{
Transport-Limited Denudation Regime Inferred from Sand Petrography and Chemical Composition: Cenozoic Sediments from the Guadiana Basin (SW Spain)
}

\author{
José Arribas', Meaza Tsige ${ }^{2}$, Guillermina Garzón², Rosa Tejero ${ }^{2}$ \\ ${ }^{1}$ Departamento de Petrología y Geoquímica, Facultad de CC. Geológicas, Universidad Complutense de Madrid, \\ Madrid, Spain \\ ${ }^{2}$ Departamento de Geodinámica, Facultad de CC. Geológicas, Universidad Complutense de Madrid, Madrid, \\ Spain \\ Email: arribas@ucm.es, meaza@ucm.es, $\underline{\text { minigar@ucm.es, rosatej@ucm.es }}$
}

Received 7 February 2014; revised 5 March 2014; accepted 3 April 2014

Copyright $@ 2014$ by authors and Scientific Research Publishing Inc.

This work is licensed under the Creative Commons Attribution International License (CC BY). http://creativecommons.org/licenses/by/4.0/

(c) (i) Open Access

\section{Abstract}

The Guadiana Basin is an E-W intraplate Cenozoic basin located in SW of the Iberian Peninsula, covering an area close to $6000 \mathrm{~km}^{2}$. NE-SW Alpine faults divide the basin into two subbasins: the Vegas Altas (VA) to the East, and the Vegas Bajas (VB) to the West. Sedimentary record consists of $<200 \mathrm{~m}$ thick of sediments, arranged in two main depositional sequences, DS1 and DS2. Petrographic analysis of sediment sandy-fraction combined with geochemical analysis of $<2 \mathrm{~mm}$ fraction permit to infer provenance and factors controlling sediment composition. Petrographic analysis of sandy-fraction reveals provenance from basin margins lithologies. In VA subbasin, quartzolithic petrofacies characterized eastern subbasin infill related to the erosion of Neoproterozoic and Palaeozoic metasediments. To the West quartzofeldspathic petrofacies predominates, associated with the erosion of coarse-grained plutonites and short distance transport of their products. Intermediate petrofacies characterizes clastic deposits from the VB. Chemical analysis of clastic deposits corroborates a main felsic origin of sediments with a composition that fits well with the upper crust compositional norm and with the Neoproterozoic Iberian Average Shale (NIBAS). In addition, minor contributions from mafic sources can be identified in DS1 from VB, manifested by anomalies in the content of $\mathrm{Co}, \mathrm{Ni}, \mathrm{Cr}, \mathrm{V}$ and Sc. Different stages of weathering (from intermediate to intense) can be deduced by the analysis of both, sandy-fraction petrography and bulk sediment geochemistry. This fact is related to the great hiatus at the base of the Cenozoicbasin infill. Transport-limited denudation regimes can be inferred by the presence of high and intermediate weathered and texturalyinmature sediments. In addition, trace element ratios suggest local enrichment in dense minerals, attributable to recycling processes from metasediments. Finally, the sedimen- 
tary record of the Guadiana Basin is constituted by immature sediments formed by limited transsport processes of very variable weathered products from recycled metasedimentary and first-order sediments from granitoids.

\section{Keywords}

\section{Provenance, Geochemistry, Sediment Composition, Intraplate Basins, Denudation Regimes}

\section{Introduction}

In the $\mathrm{W}$ of the Iberian Plate, Cenozoic basins developed during the Alpine Orogeny over Variscan basement (Iberian Massif), infilling E-W elongated troughs. These basins control and lodge the main modern Atlantic river basins (Duero, Tajo and Guadiana rivers) (Figure 1).

The Guadiana Basin is located in the SW Spain, occupying their sediments an area close to $6000 \mathrm{~km}^{2}$, from Madrigalejo to Badajoz localities (Figure 1). Two geographic sectors have been classically considered: 1) Vegas Altas (VA), at the East of Mérida locality; and 2) Vegas Bajas (VB), located at the West of Mérida locality.

Sedimentary record of this basin is Palaeogene to Pliocene in age and consists of a thin pile of sediments $(<200 \mathrm{~m})$ constituted mainly by complex clastic lithologies as mixtures of gravels, sand, silt and clay particles, due to a prevalence of mass transport processes associated to alluvial fan systems ([1] [2]). Detailed stratigraphy of the basin is not well known mainly due to a discontinuous presence of outcrop hindering correlations of sedimentary units. In addition, many problems exist in relation to the origin and detailed chronology of these deposits. The great hiatus between ancient basement rocks and basinal sediment infill (at least of $220 \mathrm{Ma}$ ), suggests long periods of weathering at the source, modifying the provenance signals in sediments. Recently, two main stratigraphic sequences have been defined ([3]) and differences in clay mineralogy between those sequences were pointed ([4]).
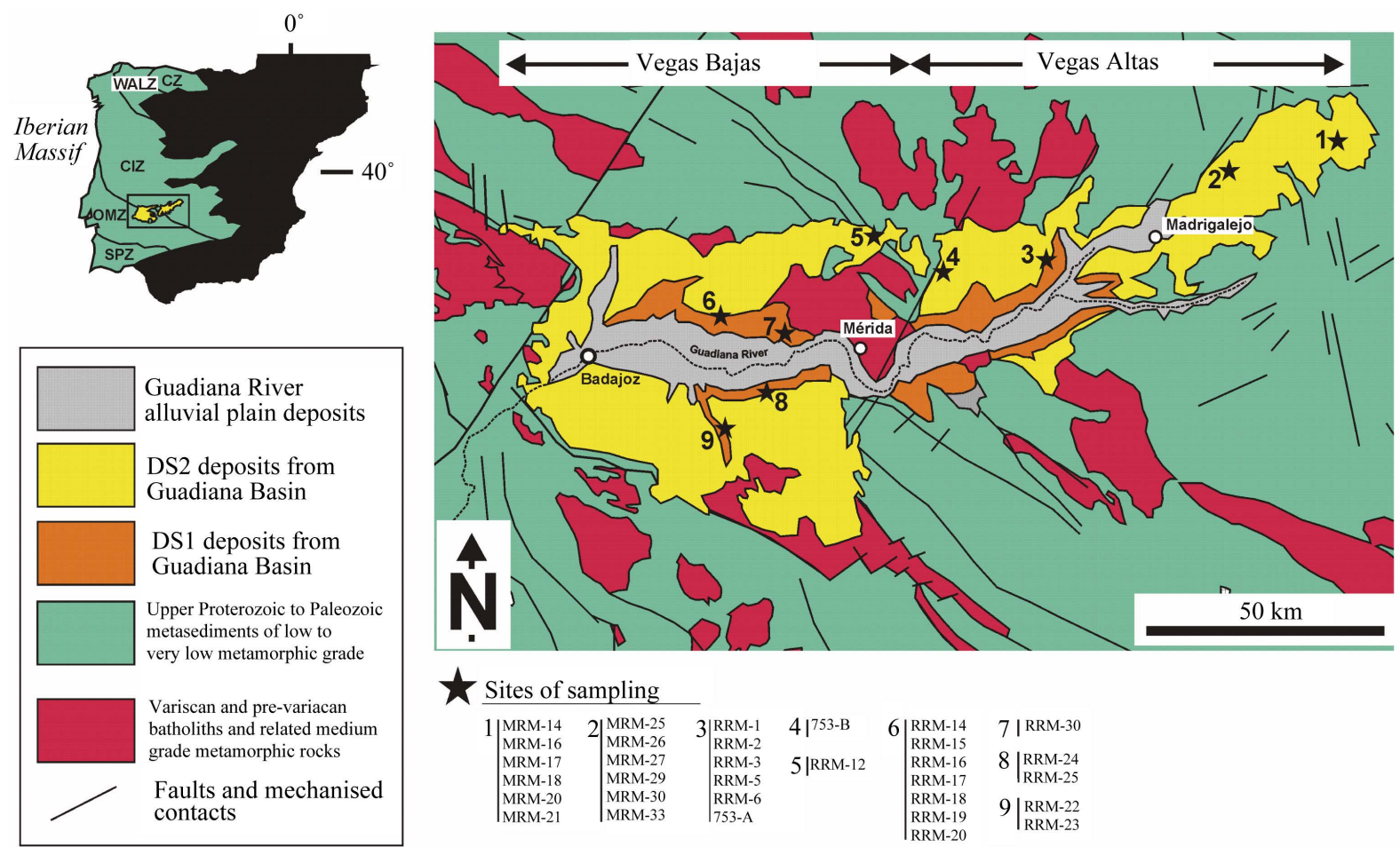

Figure 1. Location of the study area in the Iberian Peninsula and simplified geological map of the Guadiana Basin and the related substratum lithology. Location of analysed samples is also shown. Zones of the Iberian Massif: CZ, Cantabrian Zone; WALZ, Western Asturian Leonese Zone; CIZ, Central Iberian Zone; OMZ, Ossa Morena Zone; SPZ, South Portuguese Zone. 
Petrographic data from sandy deposits (e.g. [5]-[10]), together with geochemical data (e.g. [11]-[13]) has demonstrated as a very useful tools to decipher aspects related to source lithology, source weathering, transport and geotectonic setting in clastic deposits.

This paper aims to throw light to the origin and provenance of Guadiana sedimentary record, based on petrographic and geochemical analysis, and as a consequence, infer the denudation regime. These data may help in the understanding of the origin and evolution of these particular intraplate basins developing significant hiatus between basement and infill sediments.

\section{Geological Setting and Stratigraphy}

The Guadiana Basin corresponds to a Cenozoic Basin developed on the SW of the Iberian Massif. Alpine compression events in this area produced an undulatory pattern of low and highs corresponding to sedimentary basins and mountain range alignments ([14]-[16]). Thus, the Guadiana Basin is bounded by Alpine E-W folds and thrusts, and reactivated inherited left-lateral NE-SW strike-slip faults. These structures divide the basin in two small subbasins (VA and VB), (Figure 1).

The basement of the Guadiana Basin consists of Precambrian to Upper Palaeozoic metasediments and igneous rocks. Two of the major tectonostratigraphic divisions of the Hesperian Massif make up the basement of the area: the Centro Iberian Zone (CIZ), to the east, and the Ossa Morena Zone (OMZ), to the West (Figure 1). Structures present in the basement show NW-SE and E-W trends. In the CIZ, a thick succession of Upper Neoproterozoic greywackes and slates occupy the anticline cores whereas Ordovician and Silurian quarzites and slates form the interior parts of synclines ([17]). The OMZ represents the inner zone of the Variscan orogen. It includes a complete succession from Neoproterozoic to Upper Paleozoic of more than 10,000 $\mathrm{m}$ in thickness of metasediments (slates, sandstones, conglomerates and carbonates) of low to very low grade of metamorphism ([18] [19]). Locally, medium grade metamorphic lithologies (metabasites and anfibolites) are also present. Batholiths appear widespread in both zones, dominating a granitic composition. However, basic igneous rocks are also present in the OMZ, associated to the VB area of the basin.

The sedimentary record of the Guadiana Basin infill is composed of a succession of variable lithologies as red shales, sporadic thin layers of sandstones and poorly sorted quartzite and slate pebbles, gravels and arkosic sands with red and tan clay matrix. Tractive sedimentary structures are very scarce. The thickness of the succession is extremely variable (20 to $200 \mathrm{~m}$ ) and have an irregular distribution from west (VB) to east (VA) ([20] [21]). These deposits have been considered as fluvio-lacustrine sediments related to alluvial fan systems ([20]).

Based on geomorphological, sedimentological and compositional criteria, two main Depositional Sequences (DS1 and DS2) separated by a regional scale unconformity, have been distinguished ([3] [20]). This unconformity is manifested by the presence of a carbonated unit constituted by quartzite and dolomite polimictic conglomerates and dolomite layers located at the base of DS2. DS2 is finally covered by a thin layer $(<1 \mathrm{~m})$ of carbonates.

Recently, a detailed study of clay mineral composition $(<2 \mu \mathrm{m})([4])$ has revealed a different mineral association for the two depositional sequences. DS1 is characterized by an illite (predominant)-kaolinite-smectite assemblage. By contrast, clastic sediments from DS2 exhibit a smectite (predominant)-illite-kaolinite mineral assemblage. In addition, carbonated levels at the base of DS2 show a dolomite-palygorskite assemblage, whereas carbonates from top of DS2 are characterized by a calcite-palygorskite assemblage.

\section{Methods}

A total of 32 selected samples of clastic and carbonate deposits were collected from the two depositional sequences (DS1 and DS2) in both VA and VB subbasins from the Guadiana Basin. A group of 11 samples were used to extract the medium sand fraction $(0.25-0.5 \mathrm{~mm})$ by wet sieving to be investigated following classic methods in petrographic analysis (e.g. [5] [6]). Thin sections were performed on artificially cemented medium sandy-fraction with epoxy resin. Selective feldspar stain methods were used to facilitate mineral identification ([22]). Four hundred grains were counted per thin section to achieve statistical data about the distribution of the main petrographic classes of detrital grains (Table 1). Ambivalent point counting method and petrographic criteria from ([6] [23]) have been used to elaborate the petrographic data set for medium-grained sandy-fraction (Table 1).

Finally, chemical analysis of the $<2 \mathrm{~mm}$ fraction was carried out on 32 selected samples, avoiding gravel 
Table 1. Petrographic data base of medium $(0.25-0.5 \mathrm{~mm})$ sandy-fraction of selected samples from the Guadiana Basin. NCE, non-carbonate extrabasinal; NCI, non-carbonate intrabasinal; CE, carbonate extrabasinal; CI, carbonate intrabasinal; Q, total quartz; K, K-feldspar; P, plagioclase; M, mica; L, lithics. Parameters and indices are based on criteria from ([5] [23] [24] [36] [39]). N: number of counted points.

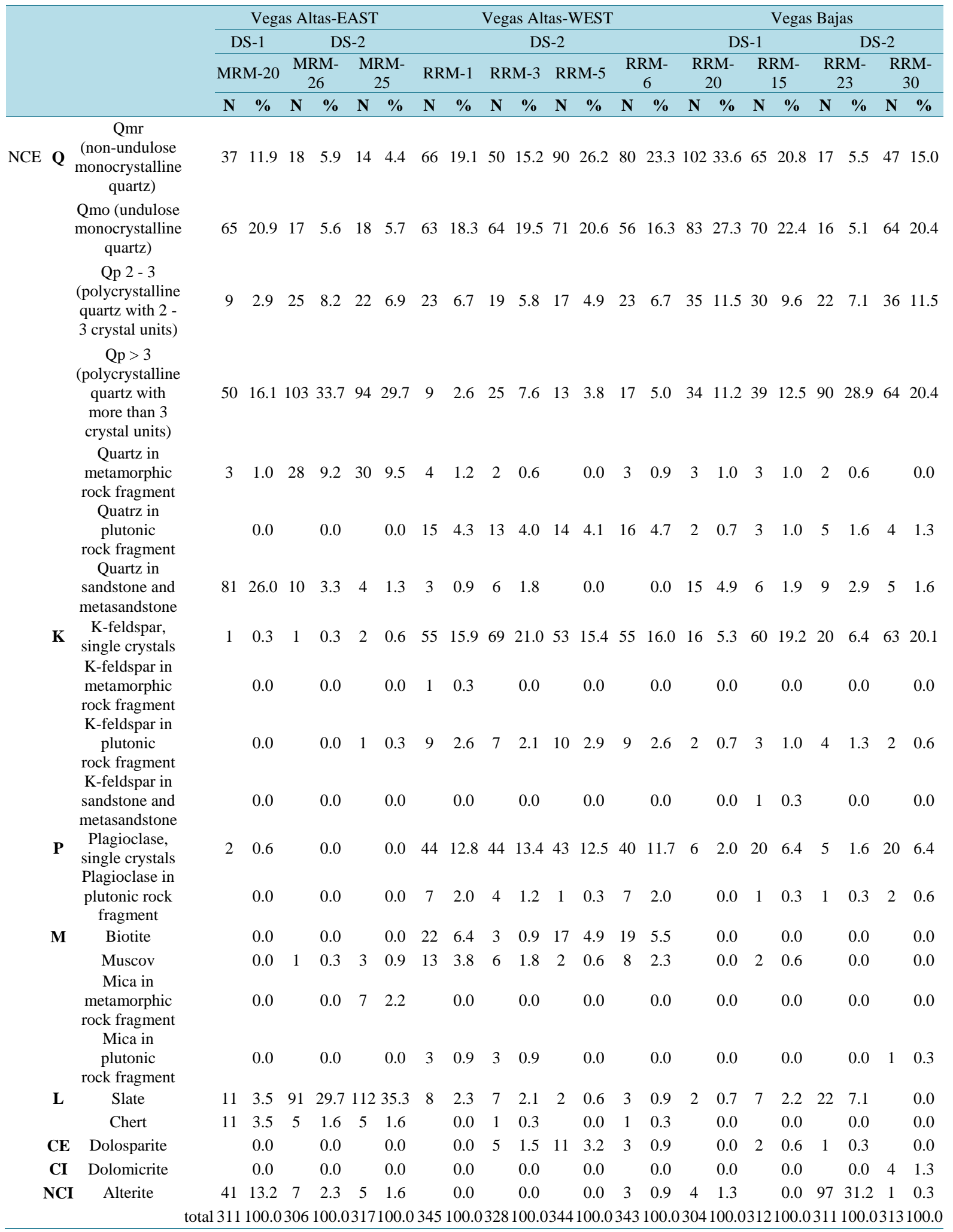




\begin{tabular}{|c|c|c|c|c|c|c|c|c|c|c|c|c|}
\hline \multicolumn{13}{|l|}{ Continued } \\
\hline \multirow{12}{*}{$\begin{array}{l}\text { PETROGRAPHIC } \\
\text { INDICES }\end{array}$} & $\mathrm{Q}$ & 63.7 & 56.4 & 49.5 & 51.9 & 49.8 & 58.8 & 56.5 & 84.7 & 65.8 & 67.8 & 68.5 \\
\hline & $\mathrm{F}$ & 1.1 & 0.3 & 0.6 & 32.3 & 35.4 & 29.5 & 30.4 & 7.3 & 25.8 & 11.7 & 26.9 \\
\hline & $\mathrm{R}$ & 35.2 & 43.3 & 49.8 & 16.1 & 14.7 & 11.7 & 13.1 & 8.0 & 8.4 & 20.6 & 4.5 \\
\hline & Qmr & 23.0 & 11.0 & 9.5 & 41.0 & 31.6 & 47.1 & 45.5 & 40.2 & 31.9 & 11.7 & 22.3 \\
\hline & Qmo & 40.4 & 10.4 & 12.2 & 39.1 & 40.5 & 37.2 & 31.8 & 32.7 & 34.3 & 11.0 & 30.3 \\
\hline & Qp & 36.6 & 78.5 & 78.4 & 19.9 & 27.8 & 15.7 & 22.7 & 27.2 & 33.8 & 77.2 & 47.4 \\
\hline & Qm & 90.7 & 67.2 & 58.3 & 91.3 & 69.2 & 53.0 & 54.6 & 59.6 & 57.4 & 75.2 & 71.4 \\
\hline & $\mathrm{F}$ & 1.1 & 0.3 & 1.0 & 8.0 & 27.2 & 33.6 & 37.8 & 31.1 & 32.6 & 14.0 & 28.2 \\
\hline & $\mathrm{Lt}$ & 8.1 & 32.1 & 37.5 & 0.7 & 2.9 & 2.3 & 4.0 & 3.8 & 2.1 & 10.7 & 0.0 \\
\hline & $\mathrm{Qm}$ & 98.8 & 99.5 & 98.4 & 61.2 & 59.1 & 65.7 & 63.7 & 91.9 & 71.8 & 84.3 & 71.7 \\
\hline & K & 0.4 & 0.5 & 1.6 & 21.7 & 25.1 & 20.2 & 20.9 & 6.0 & 21.3 & 12.6 & 21.2 \\
\hline & $\mathrm{P}$ & 0.8 & 0.0 & 0.0 & 17.1 & 15.8 & 14.1 & 15.4 & 2.0 & 7.0 & 3.1 & 7.2 \\
\hline
\end{tabular}

fraction to obtain a set of homogeneous and contrastable data. Major and trace elements were determined for all samples. Analyses were performed at Actlabs Laboratories (Canada) following Code 4Lithoresearch. Geochemical data are reported in Table 2, Table 3 and Table 4.

\section{Petrography of Sand}

\subsection{Grain Types}

Following criteria in ([24]), sand grain population is mainly constituted by non-carbonate extrabasinal (NCE) grains. Locally, sparitic carbonate extrabasinal (CE) grains have been identified. Quartz is the main detrital component exhibiting a wide spectrum of types (monocrystalline, polycrystalline and chert). Feldspars population, if present, is dominated by K-feldspars over plagioclases. Rock fragments are mainly fine-grained low grade metamorphic (shales), and quartzose metasandstones. Frequently, quartzofeldspatic coarse-grained rock fragments (plutonic) appears as a significant detrital population.

\subsection{Petrofacies}

Petrographic composition of medium sand fraction is highly variable, as arkosic, subarkosic, litharenitic and sublitharenitic compositions (Figure 2(a)). Two main petrofacies have been defined: Petrofacies A and B.

Petrofacies A, exhibits a litharenitic composition, with a quartz grain content greater than $50 \%$ and a lithic population that varies from $8 \%$ to $38 \%$, dominated by low grade metamorphic fragments (fine-grained shales). Quartzose coarse-grained rock fragments (metasandstone) are very common.Quartz population is mainly constituted by polycrystalline and undulatory monocrystalline types (Figure 2(b)). Chert and alterite grains (rounded Fe-oxidiced clasts) are also present. Feldspars are absent or appear as trace amounts. This petrofacies characterizes the eastern part of the VA subbasin (VAE in Figure 1 and Figure 2(a)).

Petrofacies B, is constituted by a quartzofeldespathic composition with a prevalence of K-feldspar over plagioclase (Figure 2(d)). Quartz grain population (60\% to 90\%) consists mainly by monocrystalline types. Rock fragments are scarce $(<20 \%)$ and are mainly coarse-grained quartzofeldspathic (plutonic) grains. Low-grade metamorphic fine-grained lithics (shales) have been also identified. Locally, monocrystalline and polycrystalline calcite and minor dolomite grains are also common. This petrofacies B characterizes the western area of VA and the entire sedimentary record in VB (VAW in Figure 1 and Figure 2(a)).

Intermediate petrofacies between A and B frequently develop in VB. This intermediate petrofacies shows a greater quartz population where the amount of polycrystalline types increases noticeably (Figure 2(b)).

\section{Geochemical Composition}

\subsection{Grain Size and Geochemical Composition}

Grain size is a significant factor that produces variations in chemical composition from the bulk clastic sediments. 
Table 2. Content in fine-fraction $(<0.062 \mathrm{~mm}$ ), major element composition (in weight \%) and related parameters of samples from the Guadiana Basin. A, CN, K and CIA parameters from ([11]).

\begin{tabular}{|c|c|c|c|c|c|c|c|c|c|c|c|c|c|c|c|c|c|}
\hline \multirow[b]{2}{*}{ Dep. Sequence } & \multirow[b]{2}{*}{ Sample } & \multirow{2}{*}{$\begin{array}{c}\% \\
<0.062 \\
\mathrm{~mm}\end{array}$} & \multirow{2}{*}{$\mathrm{SiO}_{2}$} & \multicolumn{6}{|c|}{ MAJOR ELEMENTS } & \multirow[b]{2}{*}{$\mathrm{K}_{2} \mathrm{O}$} & \multirow[b]{2}{*}{$\mathrm{TiO}_{2}$} & \multirow[b]{2}{*}{$\mathbf{P}_{2} \mathbf{O}_{5}$} & \multirow[b]{2}{*}{${ }_{5}$ LOI } & \multicolumn{4}{|c|}{ PARAMETERS } \\
\hline & & & & $\mathbf{A l}_{2} \mathbf{O}_{3}$ & $\mathrm{Fe}_{2} \mathrm{O}_{3}$ & MnO & MgO & $\mathrm{CaO}$ & $\mathrm{Na}_{2} \mathrm{O}$ & & & & & A & CN & $\mathbf{K}$ & CIA \\
\hline DS-1 VA & MRM-20 & 25.0 & 63.57 & 15.57 & 8.48 & 1.021 & 0.39 & 0.03 & 0.11 & 2.10 & 0.936 & 0.09 & 6.84 & 0.153 & 0.002 & 0.022 & 86.44 \\
\hline DS-1 VA & MRM-21 & 80.0 & 67.55 & 15.78 & 5.88 & 0.044 & 0.38 & 0.04 & 0.12 & 1.94 & 1.076 & 0.06 & 6.00 & 0.155 & 0.003 & 0.021 & 86.59 \\
\hline \multirow[t]{2}{*}{ DS-1 VA } & MRM-30 & 25.5 & 66.37 & 13.58 & 7.10 & 0.085 & 2.16 & 0.32 & 0.73 & 2.32 & 0.828 & 0.09 & 6.56 & 0.133 & 0.017 & 0.025 & 76.00 \\
\hline & & & 65.83 & 14.98 & 7.15 & 0.383 & 0.98 & 0.13 & 0.32 & 2.12 & 0.947 & 0.08 & 6.47 & 0.147 & 0.007 & 0.023 & 83.05 \\
\hline DS-1 VB & RRM-14 & 77.8 & 44.86 & 17.94 & 7.16 & 0.069 & 3.48 & 4.86 & 0.36 & 3.42 & 0.578 & 0.17 & 17.08 & 0.176 & 0.092 & 0.036 & 57.89 \\
\hline DS-1 VB & RRM-15 & 10.6 & 70.03 & 11.61 & 3.37 & 0.023 & 1.50 & 0.50 & 1.09 & 2.32 & 0.515 & 0.06 & 7.16 & 0.114 & 0.027 & 0.025 & 68.67 \\
\hline DS-1 VB & RRM-16 & 56.0 & 42.15 & 17.56 & 7.02 & 0.074 & 5.33 & 4.45 & 0.20 & 3.59 & 0.543 & 0.15 & 19.79 & 0.172 & 0.083 & 0.038 & 58.70 \\
\hline \multirow[t]{2}{*}{ DS-1 VB } & RRM-20 & 13.0 & 72.05 & 11.49 & 3.82 & 0.053 & 0.66 & 0.63 & 0.70 & 2.02 & 0.892 & 0.16 & 6.64 & 0.113 & 0.023 & 0.021 & 71.97 \\
\hline & & & 57.27 & 14.65 & 5.34 & 0.055 & 2.74 & 2.61 & 0.59 & 2.84 & 0.632 & 0.14 & 12.67 & 7 0.144 & 0.056 & 0.030 & 62.50 \\
\hline DS-2 VA & MRM-17 & 97.2 & 52.01 & 13.39 & 4.67 & 0.077 & 4.86 & 3.80 & 0.15 & 2.44 & 0.788 & 0.08 & 16.04 & 40.131 & 0.070 & 0.026 & 57.71 \\
\hline DS-2 VA & MRM-18 & $*$ & 37.35 & 11.24 & 3.96 & 0.111 & 6.41 & 12.82 & 0.13 & 2.13 & 0.572 & 0.10 & 23.88 & 0.110 & 0.231 & 0.023 & $*$ \\
\hline DS-2 VA & MRM-25 & 51.4 & 62.95 & 14.25 & 7.05 & 0.070 & 2.58 & 0.31 & 0.84 & 2.38 & 1.271 & 0.08 & 7.18 & 0.140 & 0.019 & 0.025 & 76.09 \\
\hline DS-2 VA & MRM-26 & 24.9 & 63.43 & 13.42 & 6.49 & 0.059 & 2.74 & 0.49 & 0.73 & 2.13 & 0.896 & 0.17 & 7.90 & 0.132 & 0.021 & 0.023 & 75.00 \\
\hline DS-2 VA & MRM-27 & 67.9 & 57.14 & 12.91 & 6.19 & 0.069 & 3.95 & 3.15 & 0.66 & 1.92 & 0.967 & 0.13 & 11.70 & 0.127 & 0.067 & 0.020 & 59.35 \\
\hline DS-2 VA & MRM-29 & 50.5 & 65.93 & 13.18 & 6.27 & 0.050 & 2.53 & 0.34 & 0.76 & 2.06 & 1.269 & 0.07 & 6.94 & 0.129 & 0.018 & 0.022 & 76.33 \\
\hline DS-2 VA & MRM-33 & 50.9 & 71.18 & 11.99 & 4.69 & 0.144 & 1.48 & 0.30 & 1.40 & 1.75 & 1.073 & 0.13 & 6.14 & 0.118 & 0.028 & 0.019 & 71.52 \\
\hline DS-2 VA & RRM-1 & 21.5 & 54.79 & 21.23 & 4.80 & 0.092 & 1.83 & 0.89 & 1.98 & 2.75 & 0.417 & 0.07 & 10.93 & 0.208 & 0.048 & 0.029 & 72.98 \\
\hline DS-2 VA & RRM-2 & $*$ & 40.93 & 15.78 & 5.26 & 0.051 & 6.91 & 8.42 & 0.65 & 2.15 & 0.621 & 0.13 & 19.85 & 0.155 & 0.161 & 0.023 & $*$ \\
\hline DS-2 VA & RRM-3 & 26.4 & 62.26 & 19.01 & 3.00 & 0.033 & 1.26 & 1.13 & 2.09 & 3.39 & 0.629 & 0.07 & 7.04 & 0.186 & 0.054 & 0.036 & 67.39 \\
\hline DS-2 VA & RRM-5 & 57.3 & 56.93 & 16.03 & 4.30 & 0.055 & 3.05 & 2.12 & 1.40 & 2.25 & 0.683 & 0.45 & 12.37 & 70.157 & 0.060 & 0.024 & 65.15 \\
\hline DS-2 VA & RRM-6 & 18.3 & 61.25 & 16.26 & 3.64 & 0.030 & 1.91 & 1.39 & 1.69 & 2.99 & 0.317 & 0.06 & 9.52 & 0.159 & 0.052 & 0.032 & 65.43 \\
\hline DS-2 VA & 753-A & 25.0 & 59.73 & 18.20 & 3.95 & 0.031 & 1.87 & 1.98 & 1.96 & 3.13 & 0.468 & 0.16 & 9.34 & 0.179 & 0.067 & 0.033 & 64.16 \\
\hline \multirow[t]{2}{*}{ DS-2 VA } & 753-B & 52.9 & 57.71 & 15.62 & 3.59 & 0.093 & 2.57 & 2.47 & 1.05 & 2.38 & 0.756 & 0.19 & 11.70 & 0.153 & 0.061 & 0.025 & 64.02 \\
\hline & & & 57.40 & 15.18 & 4.85 & 0.069 & 3.14 & 2.83 & 1.11 & 2.42 & 0.766 & 0.14 & 11.47 & 0.149 & 0.068 & 0.026 & 61.28 \\
\hline DS-2 VB & RRM-12 & 60.0 & 63.83 & 18.73 & 3.21 & 0.032 & 0.99 & 0.60 & 1.73 & 3.14 & 0.610 & 0.09 & 7.12 & 0.184 & 0.039 & 0.033 & 71.88 \\
\hline DS-2 VB & RRM-17 & 3.4 & 66.87 & 11.66 & 4.75 & 0.069 & 2.52 & 0.98 & 1.38 & 1.78 & 1.051 & 0.06 & 9.47 & 0.114 & 0.040 & 0.019 & 65.90 \\
\hline
\end{tabular}




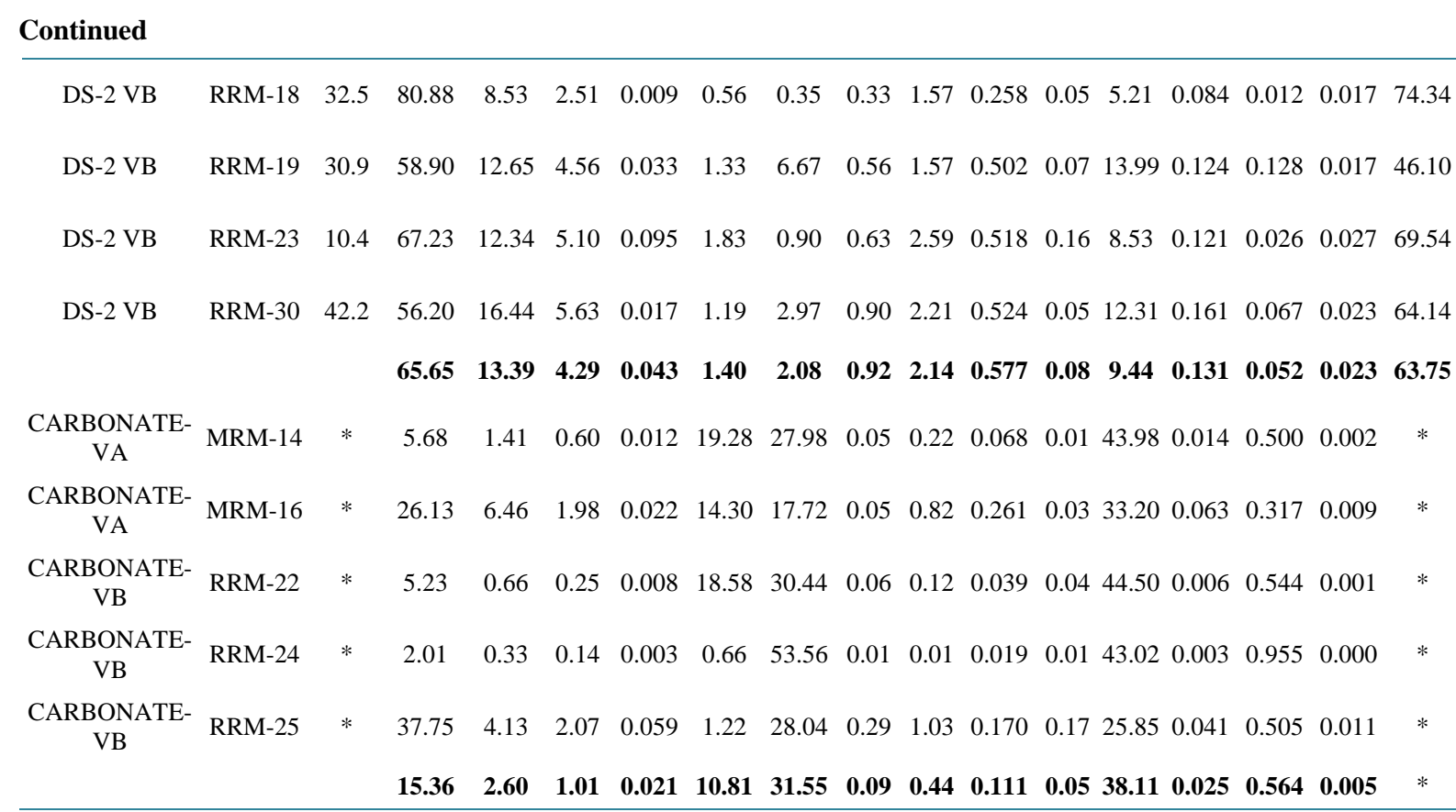

*Not considered by the high content in carbonate.
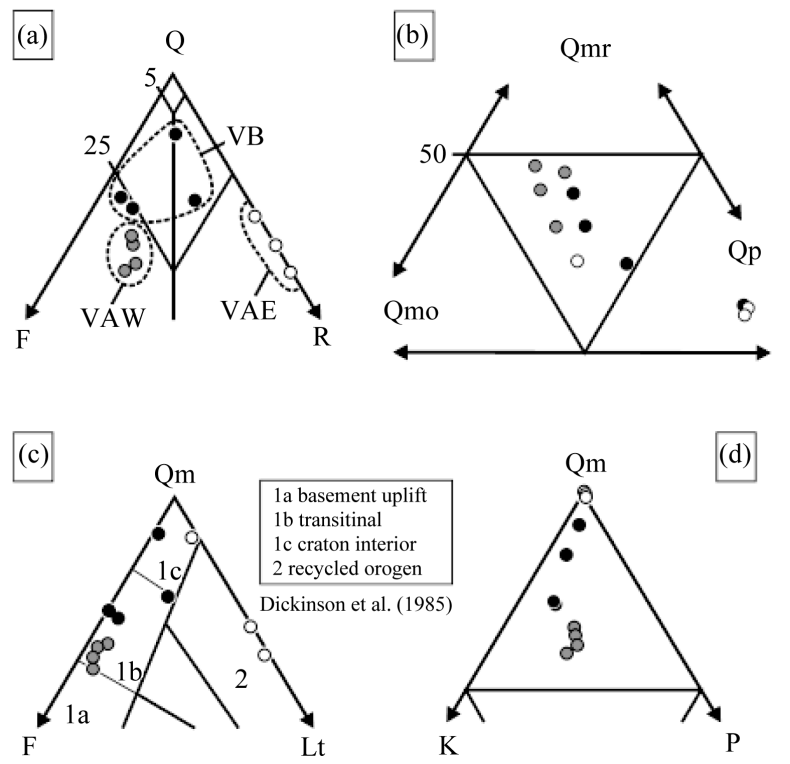

Figure 2. Ternary diagrams describing the composition of medium sandy-fraction of selected samples from the Guadiana Basin. (a) QFR diagram ([36]). VAE, samples from East of Vegas Altas; VAW, samples from the West of Vegas Altas; VB, samples from Vegas Bajas. (b): Qmr Qmo Qp diagram ([37]). (c): QmFLt diagram ([5]). (d): QmKP diagram ([5]).

The great mixing clastic size texture manifested by the wide range of fine-clastics $(<0.062 \mathrm{~mm})$ content in samples (Table 2) prevent the analysis of pure clastic intervals. However, a contrast between significant elements content $\left(\mathrm{SiO}_{2}, \mathrm{Al}_{2} \mathrm{O}_{3}, \mathrm{Na}_{2} \mathrm{O}\right.$ and Rare Earth Elements, REE) and fine-fraction content (Figure 3), reveals broad correlations. Decrease in $\mathrm{SiO}_{2}$ correlates with an increase in fine-fraction content as a function of lost of quartz and an increase of phyllosilicates in this fraction (Figure 3(a)). This last increment is also manifested by the 
Table 3. Trace element concentrations in ppm of samples from the Guadiana Basin.

\begin{tabular}{|c|c|c|c|c|c|c|c|c|c|c|c|c|c|c|c|c|c|c|c|c|c|}
\hline \multirow[b]{2}{*}{$\begin{array}{c}\text { Dep. } \\
\text { Sequence }\end{array}$} & \multirow[b]{2}{*}{ Sample } & \multicolumn{20}{|c|}{ TRACE ELEMENTS (ppm) } \\
\hline & & Ba & Co & $\mathrm{Cr}$ & Cs & Cu & Hf & $\mathbf{N i}$ & $\mathbf{P b}$ & $\mathbf{R b}$ & Sb & S & Sc & $\mathrm{Sr}$ & Ta & Th & $\mathbf{U}$ & V & $\mathbf{Y}$ & Zn & $\mathrm{Zr}$ \\
\hline DS-1 VA & MRM-20 & 2632 & 80.3 & 86.3 & 5.3 & 48 & 9.7 & 41 & 99 & 70 & 4.9 & 0.023 & 15.4 & 40 & 1.0 & 14.4 & 4.9 & 135 & 36 & 64 & 417 \\
\hline DS-1 VA & MRM-21 & 509 & 11.2 & 88.8 & 4.7 & 24 & 9.7 & 32 & 18 & 80 & 2.7 & 0.009 & 13.8 & 38 & 1.2 & 13.7 & 4.4 & 106 & 39 & 43 & 396 \\
\hline \multirow[t]{2}{*}{ DS-1 VA } & MRM-30 & 639 & 14.6 & 88.8 & 14.1 & 37 & 5.4 & 50 & 13 & 80 & 5.6 & 0.006 & 13.2 & 54 & 0.7 & 9.5 & 2.4 & 127 & 26 & 96 & 207 \\
\hline & & 1260 & 35.4 & 88.0 & 8.0 & 36 & 8.3 & 41 & 43 & 77 & 4.4 & 0.013 & 14.1 & 44 & 1.0 & 12.5 & 3.9 & 123 & 34 & 68 & 340 \\
\hline DS-1 VB & RRM-14 & 516 & 16.3 & 101.0 & 9.5 & 33 & 5.1 & 42 & 8 & 120 & 3.1 & 0.020 & 16.7 & 190 & 0.7 & 9.8 & 4.6 & 138 & 19 & 75 & 146 \\
\hline DS-1 VB & RRM-15 & 374 & 9.6 & 48.7 & 5.7 & 17 & 5.7 & 24 & 14 & 80 & 1.6 & 0.004 & 8.0 & 84 & 1.3 & 6.9 & 1.9 & 61 & 19 & 44 & 169 \\
\hline DS-1 VB & RRM-16 & 387 & 18.8 & 86.0 & 10.1 & 41 & 4.2 & 43 & 8 & 110 & 2.9 & 0.016 & 17.0 & 244 & 0.7 & 8.2 & 5.6 & 148 & 17 & 82 & 104 \\
\hline \multirow[t]{2}{*}{ DS-1 VB } & RRM-20 & 418 & 10.4 & 62.5 & 5.5 & 19 & 16.3 & 27 & 30 & 90 & 2.1 & 0.010 & 8.8 & 66 & 1.4 & 13.4 & 3.3 & 78 & 31 & 53 & 570 \\
\hline & & 424 & 13.8 & 74.6 & 7.7 & 28 & 7.8 & 34 & 15 & 100 & 2.4 & 0.013 & 12.6 & 146 & 1.0 & 9.6 & 3.9 & 106 & 22 & 64 & 247 \\
\hline DS-2 VA & MRM-17 & 499 & 12.4 & 78.8 & 5.0 & 29 & 6.1 & 33 & 21 & 90 & 2.1 & 0.007 & 11.9 & 307 & 1.1 & 11.9 & 3.2 & 83 & 24 & 62 & 236 \\
\hline DS-2 VA & MRM-18 & 364 & 12.2 & 62.2 & 4.6 & 30 & 4.1 & 29 & 20 & 80 & 1.6 & 0.008 & 10.2 & 480 & 0.8 & 9.8 & 2.8 & 68 & 19 & 54 & 151 \\
\hline DS-2 VA & MRM-25 & 603 & 16.0 & 87.3 & 12.4 & 36 & 7.8 & 53 & 18 & 80 & 4.3 & 0.004 & 13.8 & 70 & 0.9 & 11.6 & 3.1 & 128 & 32 & 96 & 366 \\
\hline DS-2 VA & MRM-26 & 531 & 13.3 & 80.7 & 12.8 & 34 & 5.2 & 49 & 9 & 70 & 3.6 & 0.004 & 12.6 & 78 & 0.7 & 9.5 & 2.2 & 115 & 32 & 92 & 216 \\
\hline DS-2 VA & MRM-27 & 549 & 14.9 & 85.1 & 11.6 & 39 & 6.1 & 49 & 13 & 70 & 3.9 & 0.005 & 12.4 & 105 & 1.0 & 9.8 & 2.4 & 111 & 26 & 80 & 248 \\
\hline DS-2 VA & MRM-29 & 488 & 12.4 & 88.1 & 11.7 & 33 & 7.2 & 45 & 18 & 80 & 4.3 & 0.004 & 12.9 & 68 & 1.4 & 11.6 & 2.9 & 118 & 32 & 92 & 312 \\
\hline DS-2 VA & MRM-33 & 569 & 25.5 & 60.6 & 3.3 & 26 & 8.8 & 68 & 15 & 50 & 3.7 & 0.009 & 10.1 & 104 & 1.0 & 11.2 & 2.7 & 83 & 30 & 67 & 370 \\
\hline DS-2 VA & RRM-1 & 603 & 9.6 & 44.2 & 6.3 & 21 & 4.5 & 31 & 15 & 100 & 0.4 & 0.003 & 10.0 & 108 & 1.0 & 7.1 & 3.5 & 63 & 23 & 72 & 134 \\
\hline DS-2 VA & RRM-2 & 516 & 12.2 & 77.1 & 5.7 & 28 & 3.9 & 26 & 15 & 90 & 1.0 & 0.014 & 14.6 & 316 & 1.4 & 12.6 & 2.7 & 125 & 21 & 46 & 100 \\
\hline DS-2 VA & RRM-3 & 1255 & 8.6 & 46.0 & 6.9 & 13 & 8.3 & 22 & 13 & 120 & 0.5 & 0.015 & 9.9 & 119 & 1.4 & 10.7 & 2.9 & 64 & 27 & 57 & 246 \\
\hline DS-2 VA & RRM-5 & 463 & 14.6 & 52.2 & 7.7 & 19 & 10.5 & 32 & 13 & 100 & 0.5 & 0.004 & 11.8 & 137 & 1.3 & 13.0 & 5.0 & 96 & 40 & 72 & 327 \\
\hline DS-2 VA & RRM-6 & 481 & 8.1 & 38.4 & 6.7 & 16 & 3.4 & 19 & 14 & 110 & 0.5 & 0.006 & 7.7 & 92 & 0.2 & 5.1 & 1.3 & 50 & 11 & 59 & 95 \\
\hline DS-2 VA & 753-A & 472 & 7.9 & 41.5 & 6.3 & 17 & 5.4 & 22 & 10 & 100 & 0.5 & 0.006 & 9.4 & 117 & 0.8 & 7.7 & 2.3 & 75 & 21 & 68 & 161 \\
\hline \multirow[t]{2}{*}{ DS-2 VA } & 753-B & 537 & 9.5 & 48.0 & 5.7 & 17 & 12.7 & 23 & 13 & 90 & 0.6 & 0.005 & 10.1 & 249 & 1.1 & 13.1 & 3.3 & 57 & 35 & 57 & 402 \\
\hline & & 566 & 12.7 & 63.6 & 7.6 & 26 & 6.7 & 36 & 15 & 88 & 2.0 & 0.007 & 11.2 & 168 & 1.0 & 10.3 & 2.9 & 88 & 27 & 70 & 240 \\
\hline DS-2 VB & RRM-12 & 582 & 7.0 & 50.8 & 6.7 & 11 & 9.1 & 23 & 17 & 110 & 1.0 & 0.005 & 9.7 & 110 & 1.2 & 13.0 & 2.8 & 74 & 26 & 43 & 298 \\
\hline DS-2 VB & RRM-17 & 398 & 14.6 & 50.3 & 4.0 & 19 & 11.4 & 30 & 21 & 60 & 2.7 & 0.011 & 9.9 & 95 & 1.8 & 7.6 & 2.7 & 96 & 24 & 54 & 378 \\
\hline
\end{tabular}




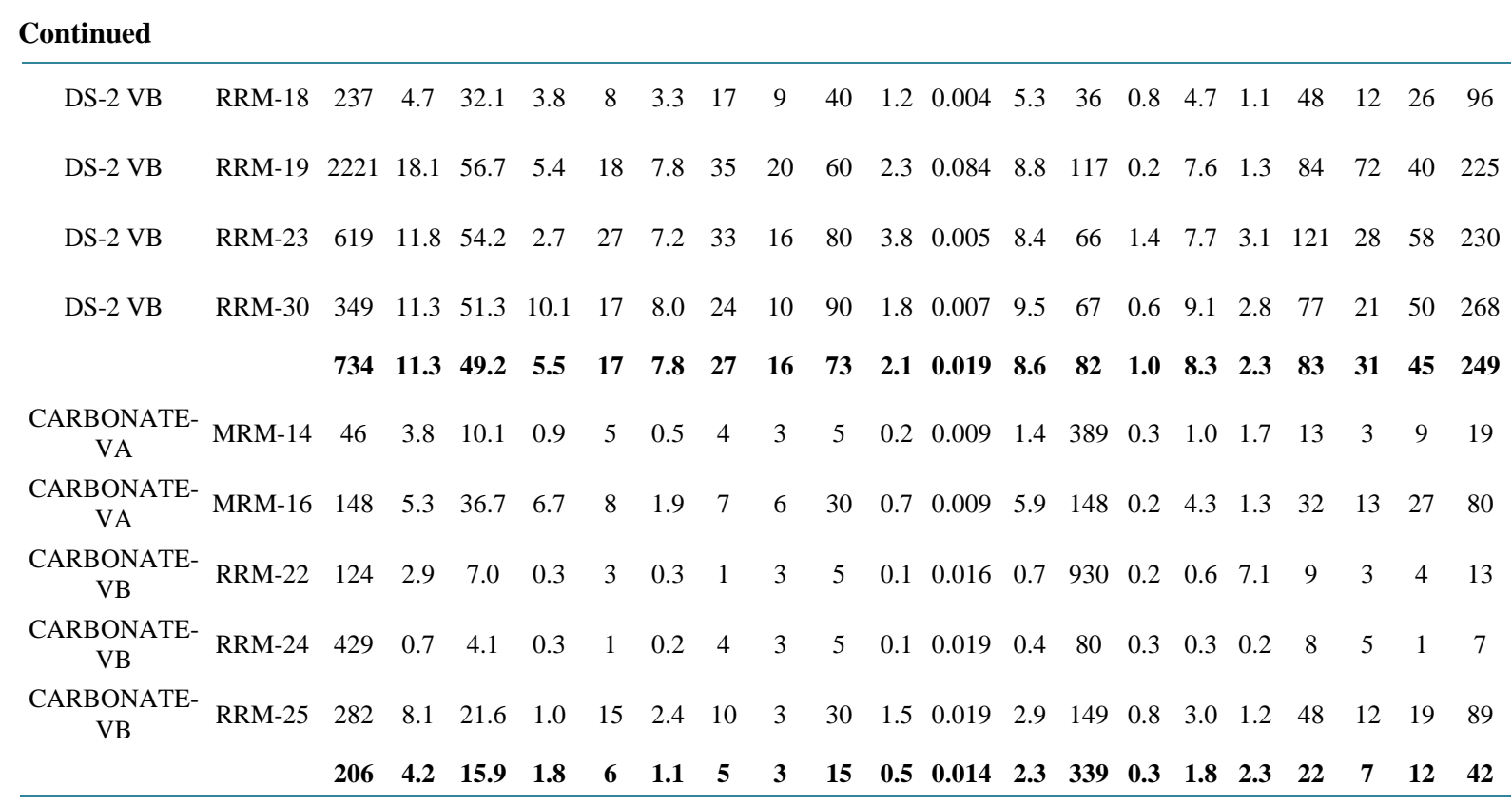

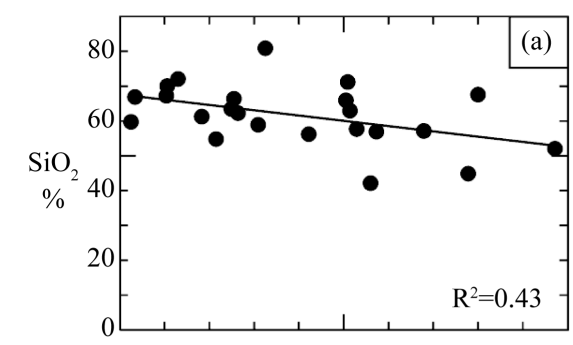

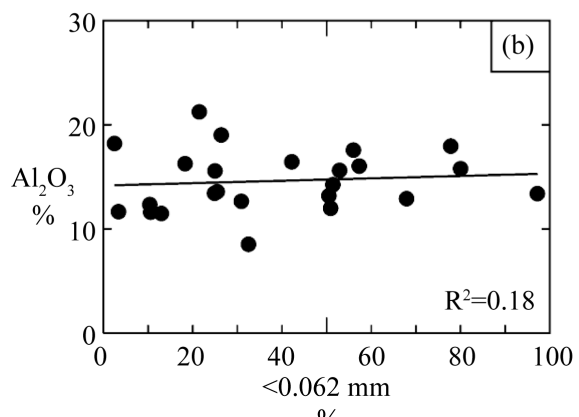

$\%$
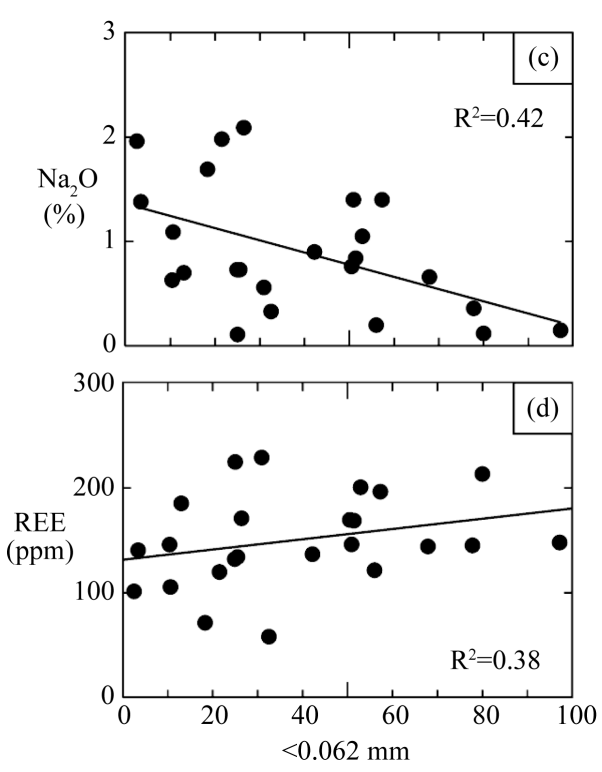

$\%$

Figure 3. Diagrams showing the correlation between the content in fine-fraction $(<0.062 \mathrm{~mm})$ and the content in different and significant chemical elements in samples from the Guadiana Basin.

slight positive correlation between $\mathrm{Al}_{2} \mathrm{O}_{3}$ and fine-fraction contents (Figure 3(b)). In addition, labile cations as $\mathrm{Na}$ are progressively removed from the sediment when fine-fraction increases (Figure 3(c)). This fact can be related to plagioclase decay. Rare Earth Element (REE) content shows positive correlation with clastic finefraction content, as a consequence of REE lodging in phyllosilicate structure (Figure 3(d)).

The low rank of correlation between composition and grain size also denotes that variations in composition are also attributable to other sedimentary processes, as nature of source and grade of weathering.

\subsection{Major Elements}

Values in $\mathrm{SiO}_{2}$ vary greatly from $37.35 \%$ to $80.88 \%$, as a result of the presence of other elements, as $\mathrm{Al}_{2} \mathrm{O}_{3}$, car- 
Table 4. Rare earth element compositions in ppm and related parameters of samples from the Guadiana Basin.

\begin{tabular}{|c|c|c|c|c|c|c|c|c|c|c|c|c|c|c|c|c|}
\hline \multirow[b]{2}{*}{$\begin{array}{c}\text { Dep. } \\
\text { Sequence }\end{array}$} & \multirow[b]{2}{*}{ Sample } & \multicolumn{8}{|c|}{ RARE EARTH ELEMENTS (REE) in ppm } & \multicolumn{7}{|c|}{ PARAMETERS } \\
\hline & & La & Ce & Nd & Sm & Eu & $\mathbf{T b}$ & $\mathbf{Y b}$ & Lu & ¿REE & LREE & HREE & $\mathbf{L a} / \mathbf{Y b}$ & $(\mathbf{L a} / \mathbf{Y b})_{N}$ & $\mathbf{L a} / \mathrm{Sm}$ & $(\mathrm{La} / \mathrm{Sm})_{\mathrm{N}}$ \\
\hline DS-1 VA & MRM-20 & 53.30 & 119 & 35 & 9.15 & 2.00 & 1.10 & 4.60 & 0.67 & 224.82 & 216.45 & 8.37 & 11.59 & 7.83 & 5.83 & 3.67 \\
\hline DS-1 VA & MRM-21 & 60.70 & 95 & 40 & 9.32 & 1.97 & 1.30 & 4.44 & 0.59 & 213.32 & 205.02 & 8.30 & 13.67 & 9.24 & 6.51 & 4.10 \\
\hline \multirow[t]{2}{*}{ DS-1 VA } & MRM-30 & 38.20 & 60 & 24 & 6.22 & 1.36 & 0.70 & 3.27 & 0.47 & 134.22 & 128.42 & 5.80 & 11.68 & 7.89 & 6.14 & 3.87 \\
\hline & & 50.73 & 91 & 33 & 8.23 & 1.78 & 1.03 & 4.10 & 0.58 & 190.79 & 183.30 & 7.49 & 12.36 & 8.36 & 6.16 & 3.88 \\
\hline DS-1 VB & RRM-14 & 31.30 & 73 & 32 & 4.65 & 1.38 & 0.05 & 2.63 & 0.30 & 145.26 & 140.95 & 4.31 & 11.90 & 8.04 & 6.73 & 4.24 \\
\hline DS-1 VB & RRM-15 & 24.30 & 53 & 20 & 3.85 & 1.22 & 0.70 & 2.26 & 0.31 & 105.64 & 101.15 & 4.49 & 10.75 & 7.27 & 6.31 & 3.97 \\
\hline DS-1 VB & RRM-16 & 27.30 & 59 & 27 & 4.39 & 1.14 & 0.05 & 2.50 & 0.26 & 121.59 & 117.69 & 3.90 & 10.92 & 7.38 & 6.22 & 3.91 \\
\hline \multirow[t]{2}{*}{ DS-1 VB } & RRM-20 & 43.90 & 90 & 39 & 5.11 & 1.70 & 1.10 & 3.96 & 0.56 & 185.33 & 178.01 & 7.32 & 11.09 & 7.49 & 8.59 & 5.41 \\
\hline & & 31.70 & 69 & 30 & 4.50 & 1.36 & 0.48 & 2.84 & 0.36 & 139.46 & 134.45 & 5.01 & 11.17 & 7.55 & 7.04 & 4.43 \\
\hline DS-2 VA & MRM-17 & 42.50 & 68 & 26 & 6.00 & 1.33 & 0.80 & 3.01 & 0.37 & 148.01 & 142.50 & 5.51 & 14.12 & 9.54 & 7.08 & 4.46 \\
\hline DS-2 VA & MRM-18 & 36.20 & 56 & 23 & 5.09 & 1.05 & 0.70 & 2.44 & 0.35 & 124.83 & 120.29 & 4.54 & 14.84 & 10.03 & 7.11 & 4.48 \\
\hline DS-2 VA & MRM-25 & 49.80 & 74 & 31 & 7.55 & 1.65 & 0.90 & 3.54 & 0.46 & 168.90 & 162.35 & 6.55 & 14.07 & 9.51 & 6.60 & 4.15 \\
\hline DS-2 VA & MRM-26 & 39.40 & 56 & 25 & 6.22 & 1.32 & 0.80 & 3.08 & 0.44 & 132.26 & 126.62 & 5.64 & 12.79 & 8.64 & 6.33 & 3.99 \\
\hline DS-2 VA & MRM-27 & 41.80 & 62 & 28 & 6.62 & 1.41 & 0.80 & 3.17 & 0.47 & 144.27 & 138.42 & 5.85 & 13.19 & 8.91 & 6.31 & 3.97 \\
\hline DS-2 VA & MRM-29 & 47.20 & 77 & 32 & 7.22 & 1.48 & 0.90 & 3.37 & 0.50 & 169.67 & 163.42 & 6.25 & 14.01 & 9.46 & 6.54 & 4.11 \\
\hline DS-2 VA & MRM-33 & 43.70 & 64 & 26 & 6.50 & 1.42 & 0.80 & 3.34 & 0.48 & 146.24 & 140.20 & 6.04 & 13.08 & 8.84 & 6.72 & 4.23 \\
\hline DS-2 VA & RRM-1 & 29.00 & 54 & 27 & 4.20 & 1.76 & 1.00 & 2.58 & 0.38 & 119.92 & 114.20 & 5.72 & 11.24 & 7.60 & 6.90 & 4.35 \\
\hline DS-2 VA & RRM-2 & 42.70 & 87 & 33 & 4.99 & 1.81 & 1.20 & 3.00 & 0.25 & 173.95 & 167.69 & 6.26 & 14.23 & 9.62 & 8.56 & 5.39 \\
\hline DS-2 VA & RRM-3 & 39.10 & 78 & 41 & 5.65 & 2.01 & 1.20 & 3.67 & 0.49 & 171.12 & 163.75 & 7.37 & 10.65 & 7.20 & 6.92 & 4.36 \\
\hline DS-2 VA & RRM-5 & 42.90 & 91 & 46 & 6.60 & 2.34 & 1.80 & 5.10 & 0.68 & 196.42 & 186.50 & 9.92 & 8.41 & 5.68 & 6.50 & 4.09 \\
\hline DS-2 VA & RRM-6 & 16.70 & 33 & 16 & 2.45 & 0.99 & 0.50 & 1.61 & 0.20 & 71.45 & 68.15 & 3.30 & 10.37 & 7.01 & 6.82 & 4.29 \\
\hline DS-2 VA & 753-A & 22.90 & 51 & 20 & 3.58 & 1.26 & 0.05 & 2.34 & 0.29 & 101.37 & 97.48 & 3.89 & 9.79 & 6.61 & 6.40 & 4.03 \\
\hline \multirow[t]{2}{*}{ DS-2 VA } & 753-B & 44.50 & 95 & 47 & 5.88 & 1.91 & 1.30 & 4.54 & 0.56 & 200.69 & 192.38 & 8.31 & 9.80 & 6.62 & 7.57 & 4.76 \\
\hline & & 38.46 & 68 & 30 & 5.61 & 1.55 & 0.91 & 3.20 & 0.42 & 147.79 & 141.71 & 6.08 & 12.02 & 8.12 & 6.85 & 4.31 \\
\hline DS-2 VB & RRM-12 & 42.10 & 83 & 42 & 5.32 & 1.91 & 1.00 & 3.43 & 0.49 & 179.25 & 172.42 & 6.83 & 12.27 & 8.29 & 7.91 & 4.98 \\
\hline DS-2 VB & RRM-17 & 29.70 & 65 & 35 & 4.56 & 1.63 & 0.80 & 3.57 & 0.43 & 140.69 & 134.26 & 6.43 & 8.32 & 5.62 & 6.51 & 4.10 \\
\hline
\end{tabular}




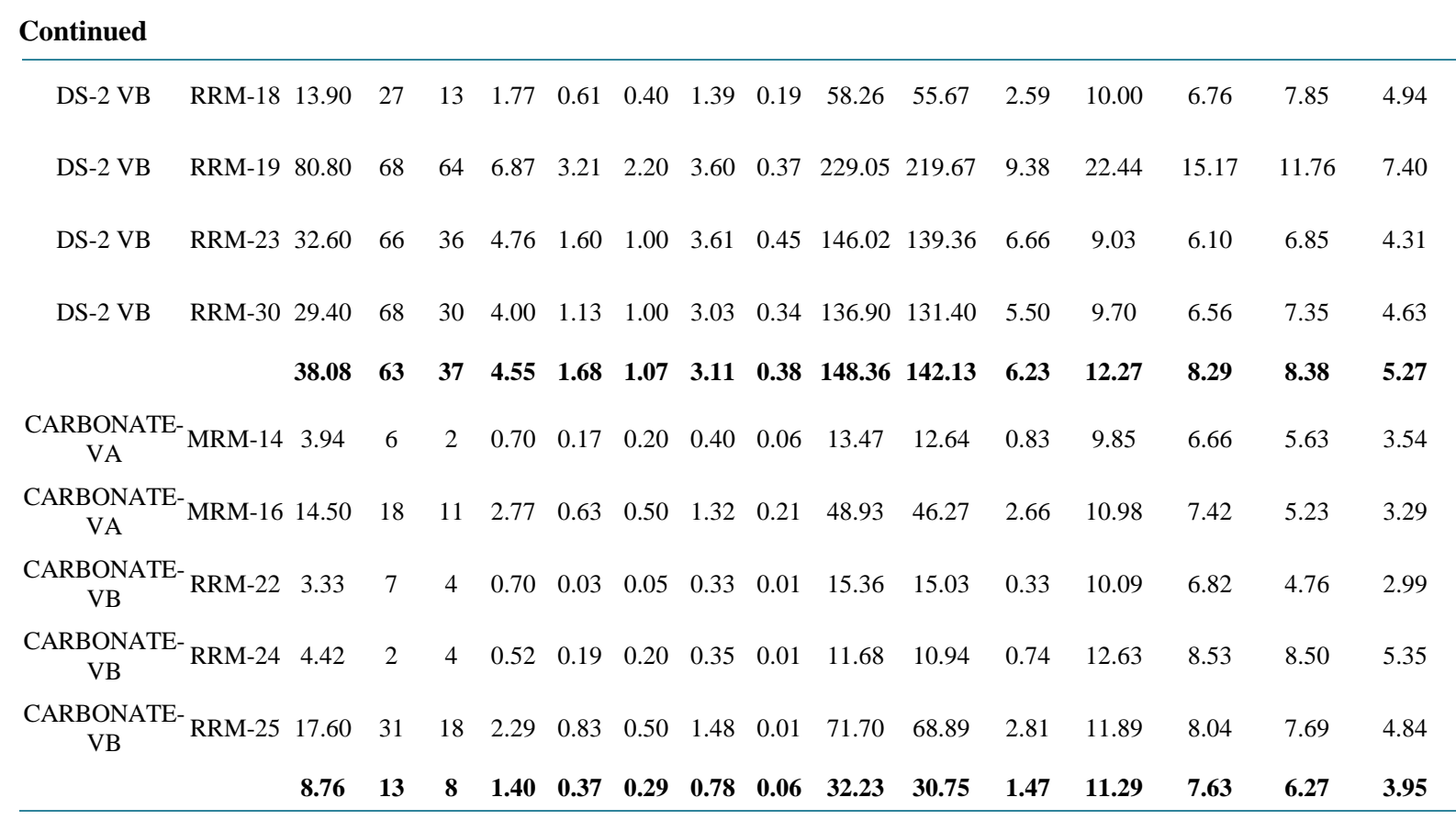

bonates $(\mathrm{CaO}, \mathrm{MgO})$, or Fe-oxides $\left(\mathrm{Fe}_{2} \mathrm{O}_{3}\right)$. Deposits dominated by the presence of sands, mainly show $\mathrm{SiO}_{2}$ values greater than 60\% (Table 2, and Figure 3(a)). By contrast, lower $\mathrm{SiO}_{2}$ contents are observed in silty-clay deposits, with higher absolute concentrations in other major elements. Thus, percentage of $\mathrm{Al}_{2} \mathrm{O}_{3}$ varies from 8.53\% to $21.23 \%$, and can be interpreted as a result of fine-fraction contents (clay minerals) (Table 2). However, high values in $\mathrm{Al}_{2} \mathrm{O}_{3}$ (greater than 15.6\%) have been also recorded in sandy deposits mainly from the western part of VA and the entire VB, associated to the quartzofeldspathic petrofacies.

$\mathrm{Fe}_{2} \mathrm{O}_{3}$ content varies from low (3.00\%) to high values (8.48\%), mainly associated to fine-grained (silty clay) detrital deposits (Table 2). Lower values $(<6 \%)$ are recorded in the DS2 from the VB.

$\mathrm{SiO}_{2}, \mathrm{Al}_{2} \mathrm{O}_{3}$ and $\mathrm{Fe}_{2} \mathrm{O}_{3}$ contents are very low in carbonate deposits from DS2. However, some samples show significant contents in $\mathrm{SiO}_{2}$, rising $37.75 \%$. This high value in silica is attributable to silicification processes, manifested by the presence of chert nodules.

The content in alkaline elements $\left(\mathrm{Na}_{2} \mathrm{O}\right.$ and $\left.\mathrm{K}_{2} \mathrm{O}\right)$ is lower than $3.4 \%$ and reflects the presence of feldspars or its weathering products (clay minerals). A very low content in $\mathrm{Na}_{2} \mathrm{O}(<1 \%)$ is observed in the sediments from the eastern part of VA, associated to the quartzolithic petrofacies. Alkaline earth elements ( $\mathrm{CaO}$ and $\mathrm{MgO})$ show variable values, from $0.56 \%$ to $6.91 \%(\mathrm{MgO})$, and from 0.03 to $12.86 \%(\mathrm{CaO})$ (Table 2). These cations show the grade of occurrence of carbonate phases in analyzed samples.

Harker diagram for $\mathrm{SiO}_{2}$ versus $\mathrm{Al}_{2} \mathrm{O}_{3}$ shows a negative correlation (Figure 4(a)). All samples fit well with this correlation if the content in other major cations (i.e., $\mathrm{CaO}$ or $\mathrm{MgO}$ ) is low. The presence of carbonate minerals in clastic deposits as detrital components or as autigenic phases (cements) reduces $\mathrm{SiO}_{2}$ and $\mathrm{Al}_{2} \mathrm{O}_{3}$ content, producing a shift of plots from the negative correlation toward the origin (Figure 4(a)). Loss On Ignition (LOI) values positively correlates with $\mathrm{CaO}$ plus $\mathrm{MgO}$, being a clear index of carbonate content in samples (Figure 4(b)).

No clear correlations (neither positive nor negative) exists between $\mathrm{SiO}_{2}$ and other major elements as $\mathrm{Fe}_{2} \mathrm{O}_{3}$, $\mathrm{K}_{2} \mathrm{O}$, MgO, $\mathrm{Na}_{2} \mathrm{O}$ or $\mathrm{TiO}_{2}$ (Table 2), suggesting that these elements are not clearly related with minerals from the sand fraction. Only a slight positive correlation exists with the content of $\mathrm{TiO}_{2}$ in samples from DS2 in VA. In this case, $\mathrm{TiO}_{2}$ content could be associated to sand sized heavy minerals.

Harker diagrams for $\mathrm{Al}_{2} \mathrm{O}_{3}$ versus $\mathrm{Na}_{2} \mathrm{O}$ and $\mathrm{K}_{2} \mathrm{O}$ show positive correlations in samples from both DS1 and DS2 (Figure 4(c) and Figure 4(d)), suggesting that these elements constitute parts of alumino-silicate minerals (feldspars, micas and clay minerals). Other elements show scatter distributions with respect $\mathrm{Al}_{2} \mathrm{O}_{3}$, as $\mathrm{MgO}$ and $\mathrm{CaO}$ suggesting a main prescence of these elements in carbonate minerals (Figure 4(e) and Figure 4(f)). Broad negative correlation between $\mathrm{Al}_{2} \mathrm{O}_{3}$ and $\mathrm{TiO}_{2}$ in DS2 samples from VA (Figure 4(g)) suggests a detrital 

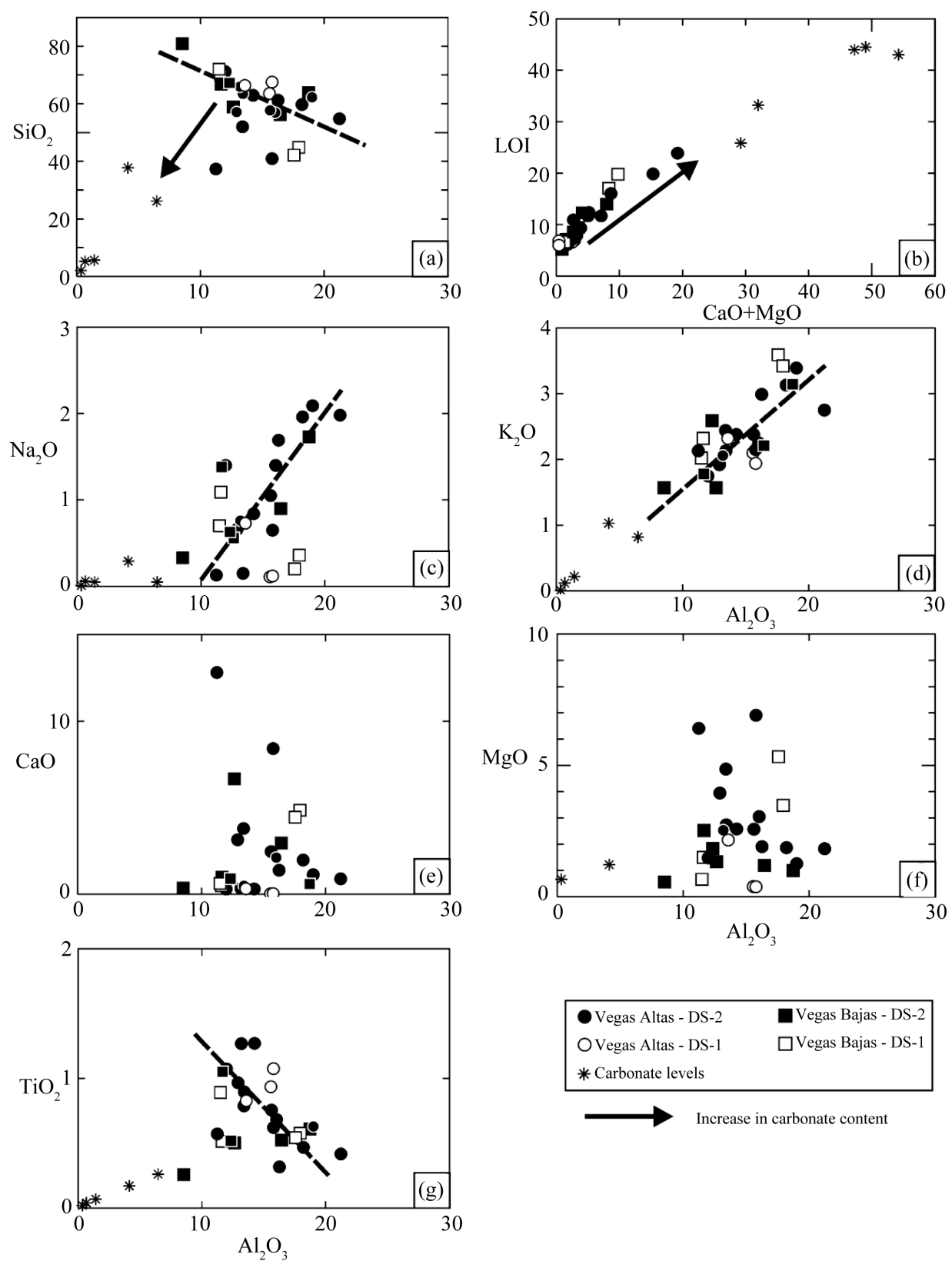

Increase in carbonate content

Figure 4. Haker diagrams showing major element variations in samples from the Guadiana Basin.

Ti-oxide as the source of this element.

\subsection{Trace Elements}

Sediment contents in Cs (mean 7 ppm), Rb (mean 85 ppm), Ba (mean 660 ppm) and Sr (mean 132 ppm) show considerable scatter values (Table 3 ) mainly as a function of grain size of the sediment. Concentration of Large Ion Lithofile Elements (LILEs) is favoured by the presence of the fine-fraction. In spite of this fact, average values are comparable with North America Shale Composite (NASC; [25]) and Average Upper Crust (AUC; [26]). A good positive correlation in $\mathrm{K}$ vs. $\mathrm{Rb}\left(\mathrm{r}^{2}: 0.85\right)$ exists. Potassium versus the other elements shows broader positive correlation, which suggests that concentration of these elements occurs mainly in K-bearing clay minerals ([13] [27] [28]). In carbonate levels, very low content in Cs (mean 2 ppm), Rb (mean 15 ppm) and Ba (mean 206 ppm) occurs. By contrast, higher concentrations in Sr are registered in these levels (mean 339 
ppm). Values of ferromagnesian trace elements contents (Co, Cr, Ni, V, and Sc) are similar to AUC ([26]). However, greater values are observed in sediments from the DS1, suggesting a slight mafic contribution from the source.

\subsection{Rare Earth Elements (REE)}

The main elements from La to Lu have been considered to obtain several parameters involving main light and heavy REE distribution (La/Yb, La/Sm, and their corresponding condrite-normalized parameters) (Table 4). Carbonate levels show very low contents in REE. No clear differences between VA and VB, neither between DS1 and DS2 sediments has been found. Thus, the analysis of the distribution of REE has been performed considering as a unique sample population of clastic and carbonate sediments (Figure 5). The most significant signatures of REE distribution in clastics are: 1) High values of $\mathrm{La} / \mathrm{Sm}$ ratio $\left((\mathrm{La} / \mathrm{Sm})_{\mathrm{N}}=\right.$ mean 4.45$)$ that suggests a steep decrease in Light Rare Earth Elements (LREE); 2) high values in La/Yb ratios $\left((\mathrm{La} / \mathrm{Yb})_{\mathrm{N}}=\right.$ mean 7.94) pointing on a flat distribution of Heavy Rare Earth Elements (HREE) (Figure 5(a)). Very low concentrations in HREE (Eu to Lu) can be deduced. As could be expected, the pattern of REE distribution is similar to that of the

Rock/Chondrites (Nakamura, 1974)
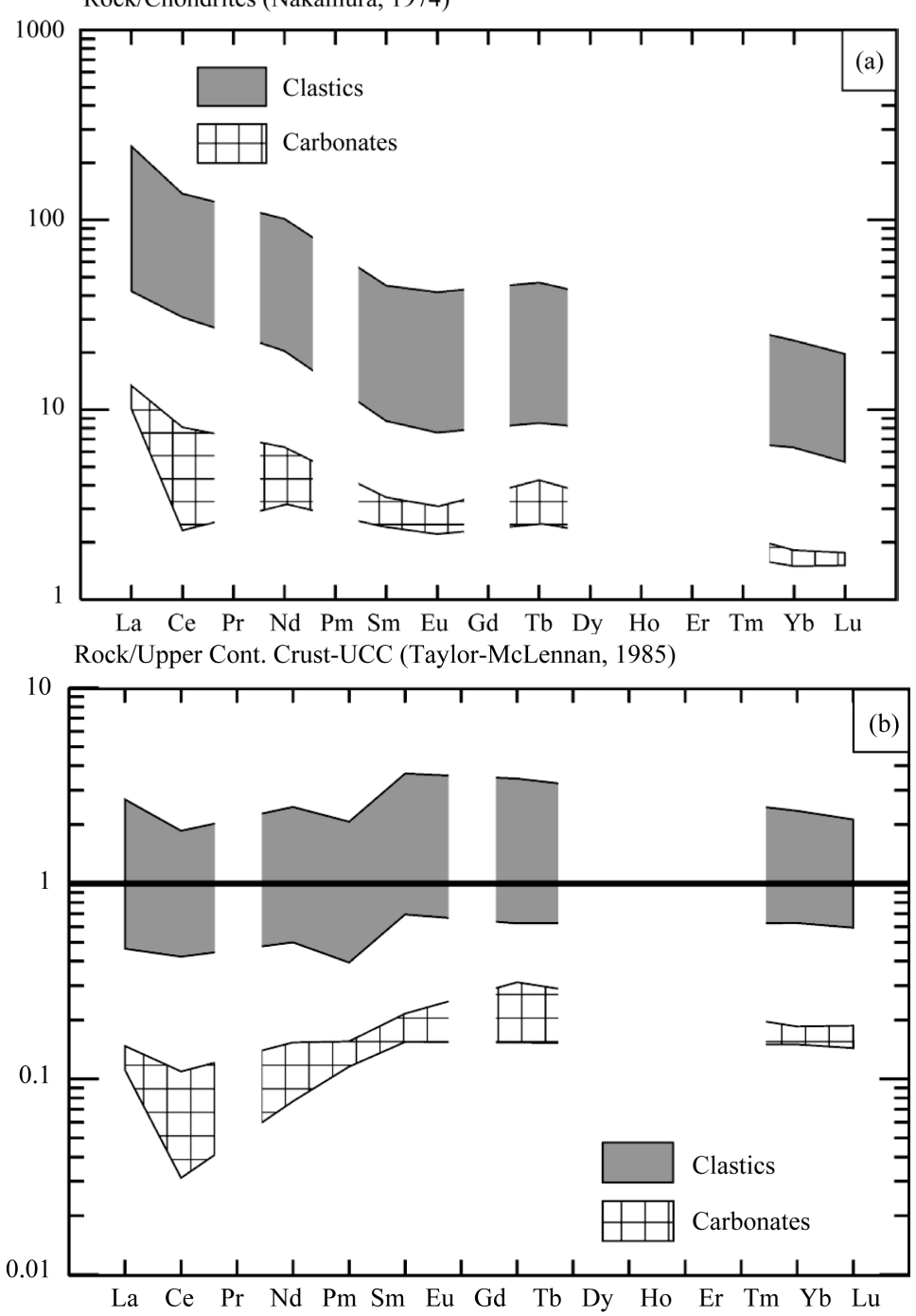

Figure 5. Diagrams showing (a) Condrite-normalized ([38]), and (b) Upper Continental Crust-normalized ([26]), of principal rare earth elements in clastic and carbonate deposits from the Guadiana Basin. The diagram shows interval values from these deposits. 
Upper Continental Crust ([26]) (Figure 5(b)).

\section{Discussion}

\subsection{Source and Tectonic Setting}

The presence of two main sandstone petrofacies in the sedimentary record of Guadiana Basin suggests significant differences concerning the lithology at the source. Quartzolithic character of petrofacies A corresponds to the supplies from the erosion of metasediments. These metasediments can be correlated with the Neoproterozoic, Cambrian and Ordovician sequences of the Central Iberian Zone from the Variscan Chain. These sedimentary sequences constitute the basement and surrounding areas of the eastern part of VA subbasin.

The quartzofeldspathic signal of petrofacies B is acquired by the erosion of plutonic terrains. The greater amount in feldspars took place in the southwestern area of the VA subbasin and can be related to the erosion of local variscan granitoids cropping close to this part of VA and northward VB. Intermediate petrofacies can be related to the mixing of petrofacies A and B and are developed mainly in DS1 and DS2 from VB.

Geochemical analysis confirms a general felsic origin of sediments that correlates with upper continental crust composition, as revealed the distribution of REE (Figure 5), and the specific La/Th vs. Hf ratios ([26] [29]). In addition, data from Guadiana Basin sediments correlate with geochemical data of Neoproterozoic Iberian Average Shale (NIBAS), in ([30]).

The spatial distribution of petrofacies can be related directly with the composition of lithologies at their sources, close to the site of deposition. This fact suggests that the sedimentary record of the basin corresponds to local supplies with very low degree of maturation. This fact is also evidenced by the low rank correlations between the bulk chemical composition and grain size (Figure 3). Thus, sediments from the eastern area of VA show a composition similar to a "recycled orogenic" provenance type ([5]) (Figure 2(c)). Western VA sediments and the main VB sediments manifest a composition similar to a "craton interior" and transition to "basement uplift” provenance types (Figure 2(c)) ([5]). Major element relationships ([31]) concur with a geotectonic setting composition associated mainly to passive continental margins (Figure 6). Quartzofeldspathic sediments from petrofacies $\mathrm{B}$ display lowest contents in $\mathrm{SiO}_{2} / \mathrm{Al}_{2} \mathrm{O}_{3}$ and $\mathrm{K}_{2} \mathrm{O} / \mathrm{Na}_{2} \mathrm{O}$ values.

\subsection{Weathering}

Intensity of weathering processes acting during the generation of sediments can be analyzed using concentration of immobile $\mathrm{Al}_{2} \mathrm{O}_{3}$ and the loss of mobile cations as $\mathrm{Ca}$, Na, and $\mathrm{K}$ ([11]) (Figure 7). Chemical Index of Altera-

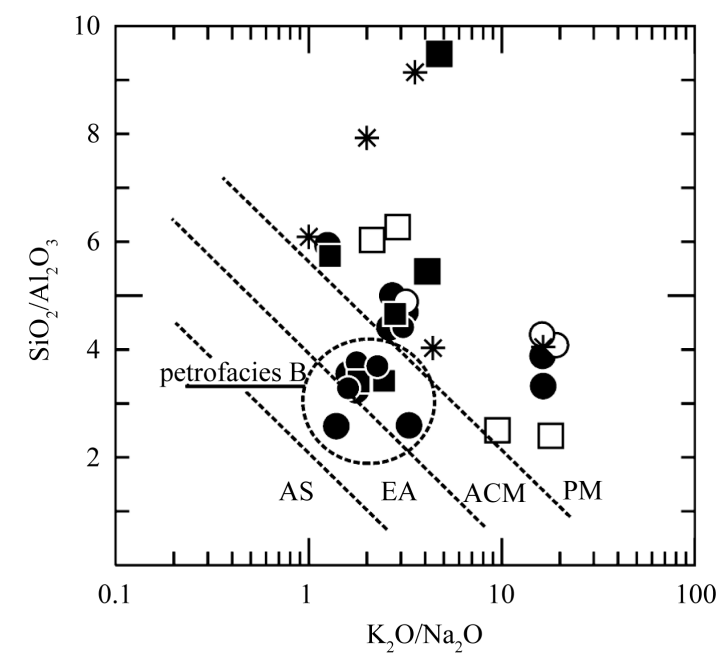

Figure 6. $\mathrm{K}_{2} \mathrm{O} / \mathrm{Na}_{2} \mathrm{O}$ versus $\mathrm{SiO}_{2} / \mathrm{Al}_{2} \mathrm{O}_{3}$ diagram ([31]) of samples from Guadiana Basin. Characteristic plot areas for several tectonic setting are shown. PM, passive margin; ACM, active continental margin; EA, evolved arc; AS, arc setting. See Figure 4 for symbols. 


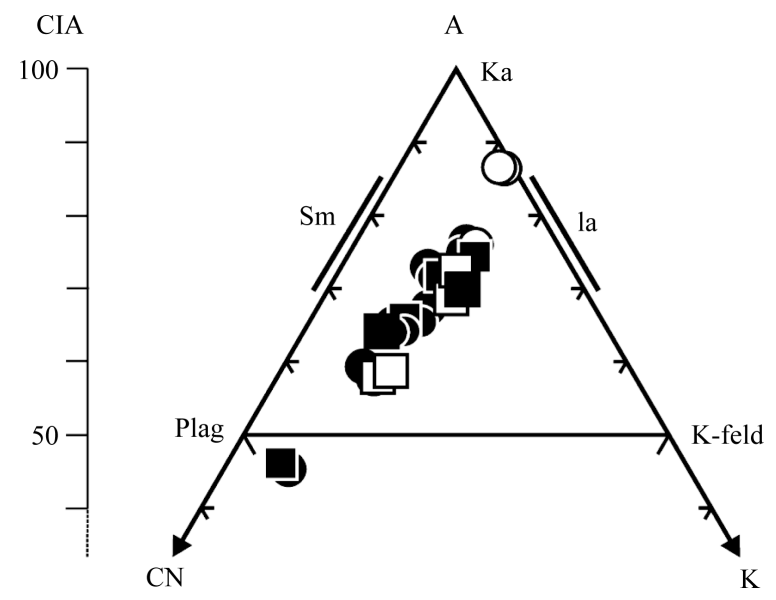

Figure 7. Chemical index of alteration (CIA) ternary plot of molecular proportions of $\mathrm{Al}_{2} \mathrm{O}_{3}(\mathrm{~A}), \mathrm{Na}_{2} \mathrm{O}+\mathrm{CaO}(\mathrm{CN})$ and $\mathrm{K}_{2} \mathrm{O}$ (K) (after [11]) for analysed samples from the Guadiana Basin. In addition, locations of some ideal composition minerals are shown. Ka, kaolinite; Sm, smectite; Ia, illite; Plag, plagioclase; K-feld, K-feldspar. See Figure 4 for symbols.

tion (CIA) values vary from close to 60 to close to 90, indicating a wide spectrum of weathered samples. Lower CIA values may correspond with samples with significant content of carbonates, increasing the content in $\mathrm{CaO}+$ $\mathrm{Na}_{2} \mathrm{O}(\mathrm{CN})$. In addition, main sample population plots following a line trend from ideal plagioclase to ideal illite composition, manifesting a progressive lost in $\mathrm{CN}$ as weathering proceeds. This trend correlates with the Zone III of a weathering profile in ([32]), and suggests a relative equilibrium between weathering and erosion at the source. Samples from DS1 at VA plot on the A-K line (Figure 7), showing a total loss of CN and a partial loss of K (CIA values close to 90). These sediments correspond to a Zone II of a weathering profile ([32]), representing a greater stage of weathering. Variation in the grade of weathering suggests changes in the type of denudation (transport limited or weathering limited, [33]). The loss of $\mathrm{Na}$ and $\mathrm{K}$ is evident if compare their contents in Cenozoic sediments with their potential Neoproterozoic sources (NIBAS in [30]). Differences in clay mineral contents between DS1 (illite dominated) and DS2 (smectite dominated) exist, suggesting changes in weathering conditions ([4]).

\subsection{Transport}

Th/Sc values close to 1 and relatively low La/Sc ratios reveal that sediments are not mature ([13] [26]) (Figure 8). In addition, significant variations in $\mathrm{Zr} / \mathrm{Sc}$ ratios with low $\mathrm{Th} / \mathrm{Sc}$ values can be interpreted as a significant enrichment of zircon in sediments ([34]) (Figure 8). This suggests that sediments have not acquired maturity during transport, but enrichment of specific dense minerals by recycling processes from metasedimentary rocks. In addition, relative contents in specific trace elements (La, Th, Co, Sc, and Zr) ([35]) show a clear fractionation of these elements due to sorting effect from sandy to silty-clay deposits (Figure 9). As expressed earlier, composition varies as a function of grain size, but sorting does not produce a clear and net compositional separation.

\section{Conclusions}

The sedimentary record that infill the intraplate Guadiana Basin (SW Spain) is a Cenozoic succession of continental, mainly siliciclastic sediments, with $<200 \mathrm{~m}$ thick. Commonly, deposits are constituted by complex textural mixtures of clasts of variable grain size (gravel, sand and mud) generated mainly by mass transport processes. These deposits outcrop in isolate areas, of difficult sedimentary correlation. Classic petrographic techniques and bulk chemical analysis (major, trace elements, and principal REE) are used to characterize these sediments and infer deductions about its genesis, nature of source areas, type of transport, and intensity of weathering, and as a consequence, the involved denudation regime.

From the petrographic analysis of the sandy-fraction, two main sources can be inferred from the presence of 

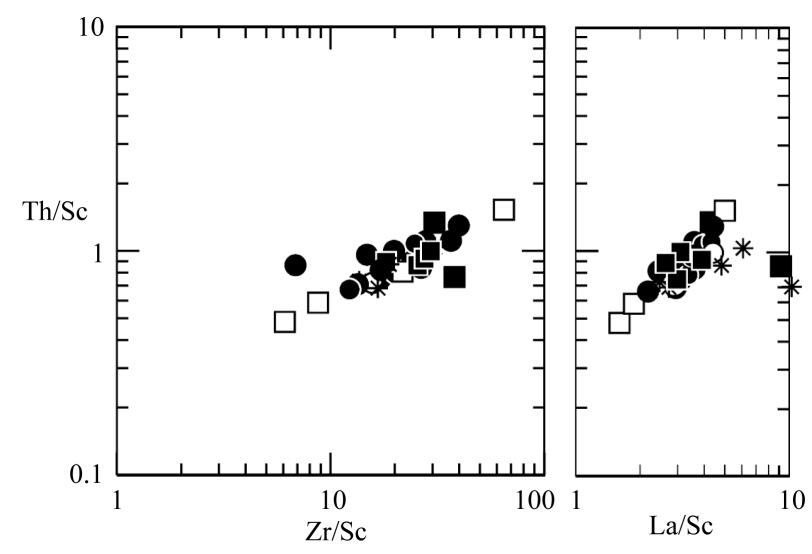

Figure 8. Th/Sc versus $\mathrm{Zr} / \mathrm{Sc}$ and $\mathrm{La} / \mathrm{Sc}$ trace element indices for analysed samples from the Guadiana Basin. See Figure 4 for symbols.
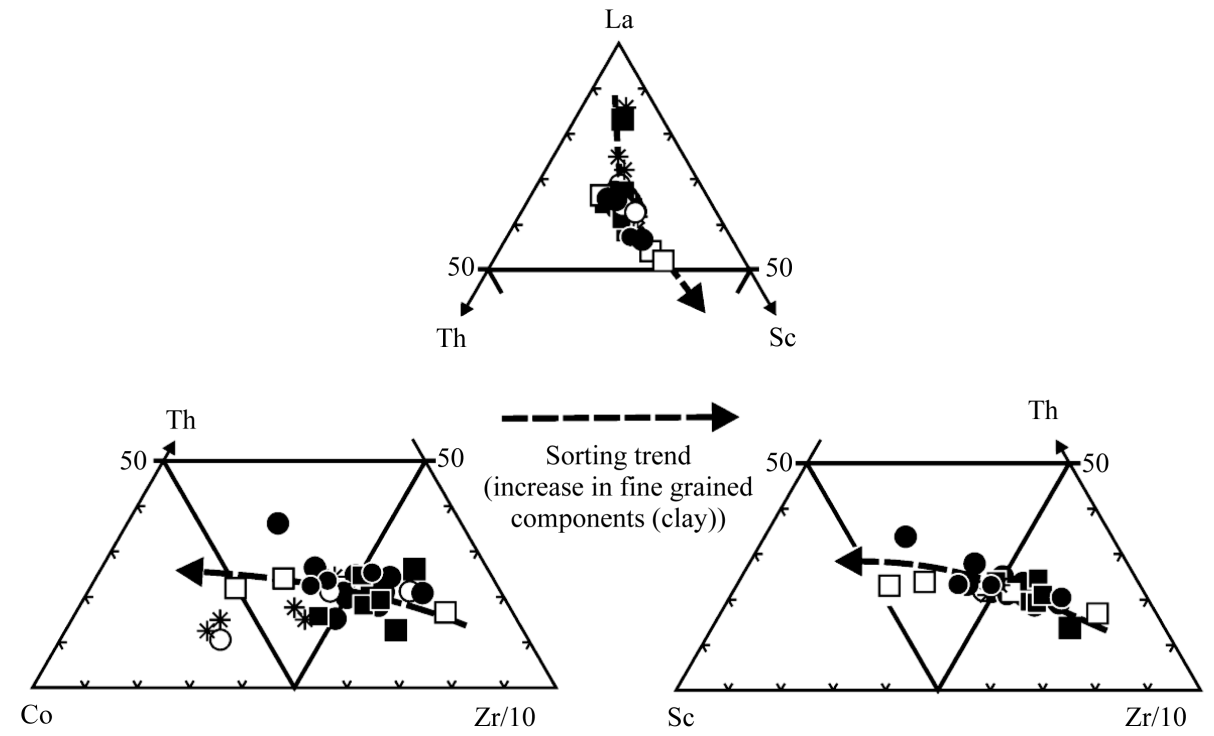

Figure 9. Trace element relations for analysed samples from the Guadiana Basin. See Figure 4 for symbols.

two petrofacies. These sources can be associated to the erosion of: 1) the Neoproterozoicand Palaeozoic succession which denotes a recycled sedimentary character, generating a quartzolithic petrofacies (petrofacies A); and 2) Coarse-grained plutonites that produces a quartzofeldspathic petrofacies (petrofacies B). Local distribution of these petrofacies in VA east and VA west, respectively, suggests short distance transport and low maturation of sediments. Intermediate petrofacies between A and B, mainly characterizes clastic deposits from the VB, as a result of mixing deposits from commented sources. Chemical analysis (including REE distribution) corroborates a main felsic origin of sediments with a composition that fits with the upper crust compositional norm and NIBAS. However, minor contributions from mafic sources can be identified in DS1 from VB (compositional anomalies in $\mathrm{Co}, \mathrm{Ni}, \mathrm{Cr}, \mathrm{V}$ and $\mathrm{Sc}$ ).

The analysis of weathering from both sandy-fraction petrography and bulk sediment geochemistry (loss of mobile cations), show different stages of weathering (from intermediate to intense), suggesting variation in its duration and intensity at the source and pointing different denudation regimes ([33]). This fact can be associated to the great hiatus at the base of the sedimentary record (DS1 in VA), being this record mainly the result of a collection of deeply weathered materials from their sources. Thus, transport-limited denudation regimes can be deduced from petrography and geochemical character of clastic deposits. A more detailed stratigraphic analysis 
is needed to establish levels of sediments from different stages of weathering.

Analysed sediments are not texturally mature by the presence of a wide range of clastic size detritus. Transport has manifested as not effective process to sort clasts. In addition, sandy composition and bulk sediment geochemistry denote low grades of compositional maturation. Several geochemical parameters show relative increases in specific dense minerals not by maturation during transport, but as a recycling process from metasedimentary sources (i.e., Neoproterozoic). Trace element fractionation can be related mainly to variations in grain size and the abundance of silty-clay fractions in sediments.

\section{Acknowledgements}

Authors thank anonymous reviewers by their suggestions and comments.This work was supported by projects CGL2011-23857 and CGL2011-22709 from the Spanish Ministry MINECO.

\section{References}

[1] Villalobos, M., Jorquera, A. and Apalategui, O. (1988) Mapa Geológico de España 1:50.000, hoja nº 802 (La Albuera). IGME, Madrid.

[2] Moreno, F., Matas, J. and Leyva, F. (2004) Mapa Geológico de España 1:50.000, hoja nº 732 (Valdecaballeros). IGME, Madrid.

[3] Garzón, G., Pellicer, M.J., Tsige, M., Tejero, R., Brum, A., Cabral, J. and Babín, R. (2012) El Cenozoico de la depresión del Guadiana en Badajoz. Nueva síntesis y propuesta de correlación. Geotemas, 13, 309-312.

[4] Tsige, M., Garzón,G., Tejero, R., Pellicer, M.J. and Babín, R. (2013) La composición mineralógica de los sedimentos terciarios de la Cuenca del Guadiana. Geogaceta, 54, 78-82.

[5] Dickinson, W.R. (1985) Provenance Relations from Detrital Modes of Sandstones. In: Zuffa, G.G., Ed., Provenance of Arenites, NATO Advanced Science Institutes Series, C-148, 333-362.

[6] Zuffa, G.G. (1985) Optical Analysis of Arenites: Influence of Methodology on Compositional Results. In: Zuffa, G.G., Ed., Provenance of Arenites, NATO Advanced Science Institutes Series, C-148, 165-190.

[7] Zuffa, G.G. (1987) Unravelling Hinterland and Offshore Palaeogeography from Deep-Water Arenites. In: Legget, J.K. and Zuffa, G.G., Eds., Marine Clastic Sedimentology, Graham \& Trotman, London, 39-61. http://dx.doi.org/10.1007/978-94-009-3241-8 2

[8] Basu, A. (1985) Influence of Climate and Relief on Compositions of Sands Released at Source Areas. In: Zuffa, G.G., Ed., Provenance of Arenites, NATO Advanced Science Institutes Series, C-148, 1-18.

[9] Arribas, J., Alonso-Millán, A., Mas, R., Tortosa, A., Rodas, M., Barrenechea, J.F., Alonso-Azcárate, J. and Artigas, R. (2003) Sandstone Petrography of Continental Depositional Sequences of an Intraplate Rift Basin: Western Cameros Basin (North Spain). Journal of Sedimentary Research, 73, 309-327. http://dx.doi.org/10.1306/082602730309

[10] Arribas, J., Ochoa, M., Mas, R., Arribas, Mª. and González-Acebrón, L. (2007) Sandstone Petrofacies in the Northwestern Sector of the Iberian Basin. Journal of Iberian Geology, 33, 191-206.

[11] Nesbitt, H.W. and Young, G.W. (1982) Early Proterozoic Climates and Plate Motions Inferred from Major Element Chemistry of Lutites. Nature, 299, 715-717. http://dx.doi.org/10.1038/299715a0

[12] McLennan, S.M., Hemming, S., McDaniel, D.K. and Hanson, G.N. (1993) Geochemical Approaches to Sedimentation, Provenance and Tectonics. In: Johnsson, M.J. and Basu, A., Eds., Processes Controlling the Composition of Clastic Sediments, Geological Society of America, Special Paper 284, 21-40. http://dx.doi.org/10.1130/SPE284-p21

[13] Gu, X.X., Liu, J.M., Zheng, M.H., Tang, J.X. and Qi, L. (2002) Provenance and Tectonic Setting of the Proterozoic Turbidites in Hunan, South China: Geochemical Evidence. Journal of Sedimentary Research, 72, 393-407. http://dx.doi.org/10.1306/081601720393

[14] Cloetingh, S., Burov, E., Beekman, F., Andeweg, B.P., Andriessen, A.M., Garcia-Castellanos, D., de Vicente, G. and Vegas, R. (2002) Lithospheric Folding in Iberia. Tectonics, 21, 1-26. http://dx.doi.org/10.1029/2001TC901031

[15] Tejero, R., Garzón Heydt, G., Babín Vich, R. and Fernández García, P. (2010) Long-Term Evolving “Tectonic” Landscapes within Intra-Plate Domains: The Iberian Peninsula. In: Veress, B. and Szigethy, J., Eds., Horizons in Earth Science Research (Vol. 2), Nova Science Publishers, Inc., USA, 103-123.

[16] Tejero, R., Garzón, G., Fernández, P., Tsige, M. and Babin, R. (2011) El control de la deformación cortical en la evolución de los relieves “tectónicos” del centro y suroeste del Macizo Ibérico. Revista de la Sociedad Geológica de España, 24, 69-84.

[17] Rodríguez Alonso, M.D., Díez Belda, M.A., Perejón, A., Pieren, A., Liñán, E., López Díaz, F., Moreno, F., Gámez Vin- 
taned, J.A., González Lodeiro, F., Martínez Poyatos, D. and Vegas, R. (2004) Dominio del Complejo Esquisto-Grauváquico. Estratigrafía. La secuencia litoestratigráfica del Neoproterozoico-Cámbrico Inferior. In: Vera, J.A., Ed., Geología de España, Sociedad Geológica de España—Instituto Geológico y Minero de España, Madrid, 78-81.

[18] Apalategui, O., Jorquera, A. and Villalobos, M. (1988) Mapa Geológico de España 1:50.000, hoja nº 801 (Olivenza). IGME, Madrid.

[19] Azor, A., Expósito, I., González Lodeiro, F., Simancas, J.F. and Martinez Poyatos, D. (2004) Zona de Ossa-Morena. Estratigrafía. Formaciones precámbricas. In: Vera, J.A., Ed., Geología de España, Sociedad Geológica de EspañaInstituto Geológico y Minero de España, Madrid, 166.

[20] Villalobos, M. and Jorquera, A. (1998) El Terciario continental y Cuaternario del sector meridional de la Cuenca del Guadiana. Publicaciones del Museo de Geología de Extremadura, 5, 33-44.

[21] Tejero López, R., Gómez Ortiz, D., Garzón Heydt, G., Tsige, M., Fernández García, P., Ortega Becerril, J.A., Babin Vich, R., Pellicer, M.J., Cabeza Dancausa, M. and Jiménez Díaz, A. (2012) La estructura de las Vegas Bajas del Guadiana. Geo-Temas, 03,127 O. CD.

[22] Chayes, F. (1952) Notes on the Staining of Potash Feldspar with Sodium Cobaltonitrite in Thinsection. American Mineralogist, 37, 337-340.

[23] Di Giulio, A. and Valloni, R. (1992) Sabbie e areniti: Analisi ottica e classificazione. Acta Naturale Ateneo Parmese, 28, $1-101$

[24] Zuffa, G.G. (1980) Hybrid Arenites: Their Composition and Classification. Journal of Sedimentary Petrology, 50, 2129.

[25] Gromet, L.P., Dymek, R.F., Haskin, L.A. and Korotev, R.L. (1984) The North American Shale Composite: Its Composition, Major and Trace Element Characteristics. Geochimica et Cosmochimica Acta, 48, 2469-2482. http://dx.doi.org/10.1016/0016-7037(84)90298-9

[26] Taylor, S.R. and McLennan, S.M. (1985) The Continental Crust: Its Composition and Evolution. Blackwell Scientific Publication, Oxford.

[27] McLennan, S.M., Taylor, S.R. and Eriksson, K.A. (1983) Geochemistry of Archean Shales from the Pilbara Supergroup, Western Australia. Geochimica et Cosmochimica Acta, 47, 1211-1222.

[28] Feng, R. and Kerrich, R. (1990) Geochemistry of Fine-Grained Clastic Sediments in the Archean Abitibi Greenstone Belt, Canada: Implications for Provenance and Tectonic Setting. Geochimica et Cosmochimica Acta, 54, 1061-1081. http://dx.doi.org/10.1016/0016-7037(90)90439-R

[29] Floyd, P.A. and Leveridge, B.E. (1987) Tectonic Environment of the Devonian Gramscatho Basin, South Cornwall: Framework Mode and Geochemical Evidence from Turbiditic Sandstones. Journal of the Geological Society, 144, 531542. http://dx.doi.org/10.1144/gsjgs.144.4.0531

[30] Ugidos, J.M., Sánchez-Santos, J.M., Barba, P. and Valladares, M.I. (2010) Upper Neoproterozoic Series in the Central Iberian, Cantabrian and West Asturian Leonese Zones (Spain): Geochemical Data and Statistical Results as Evidence for a Shared Homogenised Source Area. Precambrian Research, 178, 51-58. http://dx.doi.org/10.1016/j.precamres.2010.01.009

[31] Maynard, J.B., Valloni, R. and Yu, H.S. (1982) Composition of Modern Deepsea Sands from Arc-Related Basins. In: Leggett, J.K., Ed., Sedimentation and Tectonics on Modern and Ancient Active Plate Margins (Vol. 10), Geological Society of London, Special Publication, 551-561.

[32] Nesbitt, H.W., Fedo, C.M. and Young, G.M. (1997) Quartz and Feldspar Stability, Steady and Non-Steady-State Weathering, and Petrogenesis of Siliciclastic Sands and Muds. Journal of Geology, 105, 173-191.

[33] Johnsson, M.J. (1993) The System Controlling the Composition of Clastic Sediments. In: Johnsson, M.J. and Basu, A., Eds., Processes Controlling the Composition of Clastic Sediments, Vol. 284, Geological Society of America, Special Paper, 1-19. http://dx.doi.org/10.1130/SPE284-p1

[34] McLennan, S.M. and Taylor, S.R. (1991) Sedimentary-Rocks and Crustal Evolution: Tectonic Setting and Secular Trends. Journal of Geology, 99, 1-21. http://dx.doi.org/10.1086/629470

[35] Bhatia, M.R. and Crook, K.A.W. (1986) Trace Elements Characteristics of Greywackes and Tectonic Setting Discrimination of Sedimentary Basins. Contributions to Mineralogy and Petrology, 92, 181-193.

[36] Pettijohn, F.J., Potter, P.E. and Siever, R. (1972) Sand and Sandstone. Springer-Verlag, Berlin.

[37] Arribas, J., Alonso, A., Pagés, J.L. and González-Acebrón, L. (2010) Holocene Transgression Recorded by Sand Composition in the Mesotidal Galician Coastline (NW Spain). The Holocene, 20, 375-393. http://dx.doi.org/10.1177/0959683609353429

[38] Nakamura, N. (1974) Determination of REE, Ba, Fe, Mg, Na, and K in Carbonaceous and Ordinary Chondrites. Geochimica et Cosmochimica Acta, 38, 757-775. 
J. Arribas et al.

[39] Basu, A., Young, S.W., Suttner, L.J., James, C.W. and Mack, G.H. (1975) Re-Evaluation of the Use of Undulatory Extinction and Polycrystallinity in Detrital Quartz for Provenance Interpretation. Journal of Sedimentary Petrology, 45, 873-882. 